quatrième série - tome $46 \quad$ fascicule 2 mars-avril 2013

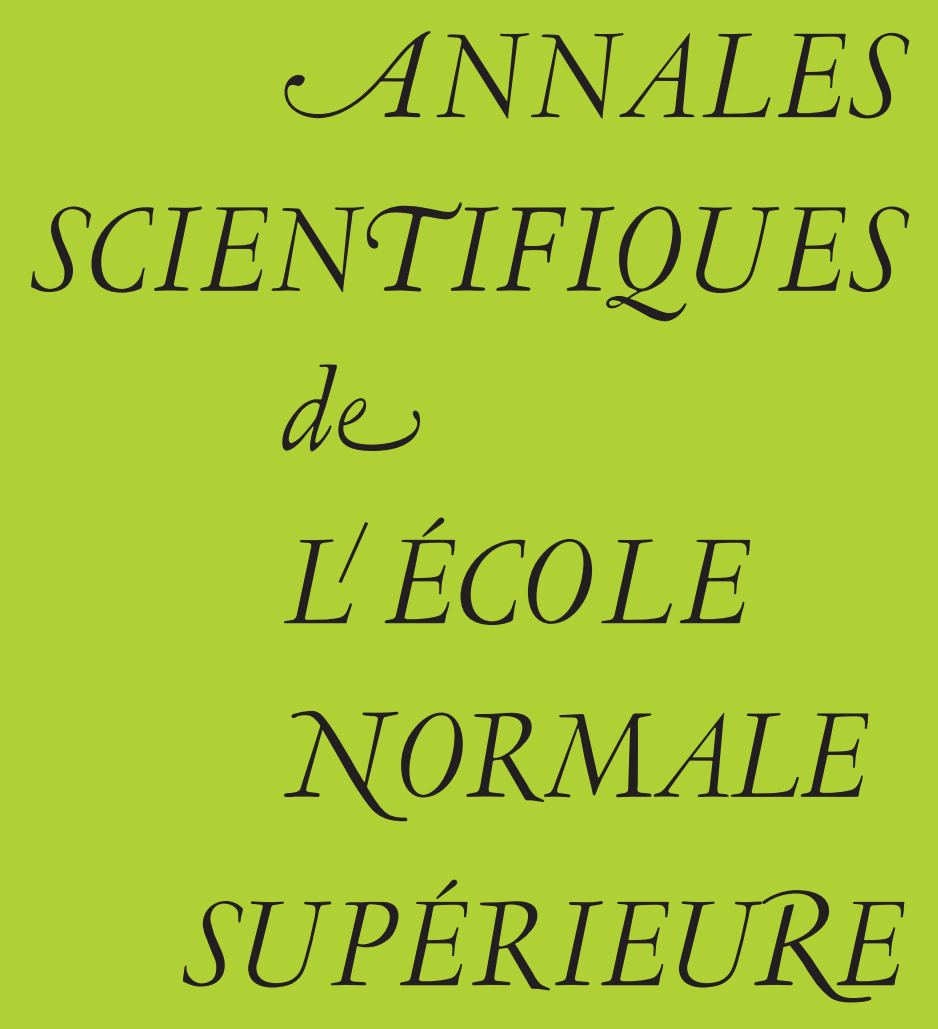

Régis MONNEAU \& Jean-Michel ROQUEJOFFRE \& Violaine ROUSSIER-MICHON

Travelling graphs for the forced mean curvature motion in an arbitrary space dimension 


\title{
TRAVELLING GRAPHS FOR THE FORCED MEAN CURVATURE MOTION IN AN ARBITRARY SPACE DIMENSION
}

\author{
BY RÉGis MONNEAU, JEAN-Michel ROQUEJOFFRE \\ AND VIOLAINE ROUSSIER-MICHON
}

Dedicated to Henri Berestycki

Abstract. - We construct travelling wave graphs of the form $z=-c t+\phi(x)$, $\phi: x \in \mathbb{R}^{N-1} \mapsto \phi(x) \in \mathbb{R}, N \geq 2$, solutions to the $N$-dimensional forced mean curvature motion $V_{n}=-c_{0}+\kappa\left(c \geq c_{0}\right)$ with prescribed asymptotics. For any 1-homogeneous function $\phi_{\infty}$, viscosity solution to the eikonal equation $\left|D \phi_{\infty}\right|=\sqrt{\left(c / c_{0}\right)^{2}-1}$, we exhibit a smooth concave solution to the forced mean curvature motion whose asymptotics is driven by $\phi_{\infty}$. We also describe $\phi_{\infty}$ in terms of a probability measure on $\mathbb{S}^{N-2}$.

RÉSUMÉ. - Nous construisons des ondes progressives sous la forme de graphes $z=-c t+\phi(x)$, $\phi: x \in \mathbb{R}^{N-1} \mapsto \phi(x) \in \mathbb{R}, N \geq 2$, solutions du mouvement par courbure moyenne forcée $V_{n}=-c_{0}+\kappa\left(c \geq c_{0}\right)$ en dimension $N$ d'espace et avec un comportement asymptotique prescrit. Pour toute solution de viscosité $\phi_{\infty}, 1$-homogène en espace, de l'équation eikonale $\left|D \phi_{\infty}\right|=\sqrt{\left(c / c_{0}\right)^{2}-1}$, nous mettons en évidence une solution régulière et concave du mouvement par courbure moyenne forcée dont le comportement asymptotique est donné par $\phi_{\infty}$. Nous décrivons aussi $\phi_{\infty}$ en terme d'une mesure de probabilité sur la sphère $\mathbb{S}^{N-2}$.

\section{Introduction}

\subsection{Setting of the problem}

The question investigated here is the description of the travelling wave graph solutions to the forced mean curvature motion in any dimension $N \geq 2$, that is written under the general form

$$
V_{n}=-c_{0}+\kappa
$$

where $V_{n}$ is the normal velocity of the graph, $\kappa$ its local mean curvature and $c_{0}$ a given strictly positive constant to be defined later. A graph satisfying (1) can be given by the equation 
$z=u(t, x)$ where $u:(t, x) \in \mathbb{R}^{+} \times \mathbb{R}^{N-1} \mapsto u(t, x) \in \mathbb{R}$ is a solution to the parabolic equation

$$
\frac{u_{t}}{\sqrt{1+|D u|^{2}}}=-c_{0}+\operatorname{div}\left(\frac{D u}{\sqrt{1+|D u|^{2}}}\right), \quad t>0, x \in \mathbb{R}^{N-1} .
$$

Indeed, at any time $t>0$ fixed, the outer normal to the subgraph $\left\{(x, z) \in \mathbb{R}^{N-1} \times \mathbb{R} \mid\right.$ $z \leq u(t, x)\}$ is given by

$$
\vec{n}=\frac{1}{\sqrt{1+|D u|^{2}}}\left(\begin{array}{c}
-D_{x} u \\
1
\end{array}\right)
$$

its normal velocity $V_{n}$ by $\left(0, \partial_{t} u\right)^{T} \cdot \vec{n}$ while its mean curvature by $\kappa=-\operatorname{div}_{(x, z)} \vec{n}$, see [6].

A travelling wave to (2) is a solution of the form $u(t, x)=-c t+\phi(x)$ where $\phi: x \in \mathbb{R}^{N-1} \mapsto \phi(x) \in \mathbb{R}$ is the profile of the wave and $c \geq c_{0}$ is some given constant standing for its speed. Thus $\phi$ satisfies the following elliptic equation

$$
-\operatorname{div}\left(\frac{D \phi}{\sqrt{1+|D \phi|^{2}}}\right)+c_{0}-\frac{c}{\sqrt{1+|D \phi|^{2}}}=0, \quad x \in \mathbb{R}^{N-1}
$$

\subsection{Connection with reaction diffusion equations}

This work should provide us a better understanding of the multidimensional solutions to the non linear scalar reaction diffusion equation

$$
\partial_{t} v=\Delta v+f(v), \quad t>0,(x, z) \in \mathbb{R}^{N-1} \times \mathbb{R}
$$

where $v:(t, x, z) \in[0,+\infty) \times \mathbb{R}^{N-1} \times \mathbb{R} \mapsto v(t, x, z) \in \mathbb{R}$ and, especially the case of travelling waves in dimension $N$. In the case of a "bistable" nonlinearity $f$, that is to say when $f$ is a continuously differentiable function on $\mathbb{R}$ satisfying

(i) $f(0)=f(1)=0$

(ii) $f^{\prime}(0)<0$ and $f^{\prime}(1)<0$

(iii) there exists $\theta \in(0,1)$ such that $f(v)<0$ for $v \in(0, \theta), f(v)>0$ for $v \in(\theta, 1)$

(iv) $\int_{0}^{1} f(v) \mathrm{d} v>0$

it is well-known [10] that there exists a one-dimensional travelling front $v(t, z)=\phi_{0}\left(z+c_{0} t\right)$ solution to (4) with $N=1$. The speed $c_{0}$ is unique and strictly positive by (iv) while the profile $\phi_{0}$ is unique up to translations. This result defines the constant $c_{0}>0$ that appears in Equation (1).

In the case $N=2$, multidimensional solutions to (4) are well understood. Paper [7] proves the existence of conical travelling waves solutions to (4), and paper [8] classifies all possible bounded non constant travelling waves solutions under rather weak conditions at infinity. In particular, it is proved in [8] that $c \geq c_{0}$ and, up to a shift in $x \in \mathbb{R}$, either $u$ is a planar front $\phi_{0}( \pm x \cos \alpha+z \sin \alpha)$ with $\alpha=\arcsin \left(c_{0} / c\right) \in\left(0, \frac{\pi}{2}\right]$ or $u$ is the unique conical front found in [7].

In higher dimensions, less is known. In [7], Hamel, Monneau and Roquejoffre or in [13], Ninomiya and Taniguchi proved the existence of conical travelling waves with cylindrical symmetry whose level sets are Lipschitz graphs moving away logarithmically from straight cones. Some special, non cylindrically symmetric pyramidal-shaped solutions (see Taniguchi [14] and references therein) are also known in any dimension $N \geq 3$. 
Thus, in order to get a better understanding of the mechanisms at work, we further the idea of bridging reaction-diffusion equations with geometric motions. In particular, travelling wave graph solutions to the forced mean curvature motion go back to Fife [5]. He proved (in a formal fashion) that reaction-diffusion travelling fronts propagate with normal velocity

$$
V_{n}=-c_{0}+\frac{\kappa}{t}+O\left(\frac{1}{t^{2}}\right), \quad t \gg 1
$$

For a mathematically rigorous treatment of these ideas, we refer for instance to de Mottoni, Schatzman [11] — small times, smooth solutions context—and Barles, Soner, Souganidis [1] - arbitrary large times, viscosity solutions context.

\subsection{Main results}

Our Theorem 1.1 below states that, given a 1-homogeneous solution $\phi_{\infty}$ to the eikonal equation derived from (3) (i.e., the equation obtained by removing the curvature term) there exists a smooth solution $\phi$ to the forced mean curvature motion Equation (3) whose asymptotic behaviour is prescribed by $\phi_{\infty}$. Here is the precise result.

Theorem 1.1 (Existence of solutions with prescribed asymptotics in dimension $N$ )

Let $N \in \mathbb{N} \backslash\{0,1\}, \alpha \in\left(0, \frac{\pi}{2}\right], c_{0}>0$ and $c=c_{0} / \sin \alpha$. Choose $\phi_{\infty}$ a 1-homogeneous viscosity solution to the eikonal equation

$$
\left|D \phi_{\infty}(x)\right|=\cot \alpha, \quad x \in \mathbb{R}^{N-1} .
$$

Then there exists a smooth concave solution $\phi \in C^{\infty}\left(\mathbb{R}^{N-1}\right)$ to (3) such that

$$
\phi(x)=\phi_{\infty}(x)+o(|x|) \text { as }|x| \rightarrow+\infty .
$$

This is the most possible general result. However, due to the possible complexity of a solution to the eikonal Equation (5), it is useful to specialize our result to the particular case of a solution with a finite number of facets.

Theorem 1.2 (Solutions with finite number of facets in dimension $N$ )

Let $N \in \mathbb{N} \backslash\{0,1\}, \alpha \in\left(0, \frac{\pi}{2}\right], c_{0}>0$ and $c=c_{0} / \sin \alpha$. Choose $\phi^{*}$ a viscosity solution to the eikonal Equation (5) given for any $x \in \mathbb{R}^{N-1}$ by

$$
\phi^{*}(x)=\inf _{\nu \in A}\left(-(\cot \alpha) x \cdot \nu+\gamma_{\nu}\right)
$$

where $A$ is a finite subset of cardinal $k \in \mathbb{N}^{*}$ of the sphere $\mathbb{S}^{N-2}$ and $\gamma_{\nu}$ are given real numbers. Then there exists a unique smooth concave solution $\phi \in C^{\infty}\left(\mathbb{R}^{N-1}\right)$ to (3) such that

$$
\left\{\begin{array}{l}
-\frac{2 \ln k}{c_{0} \sin \alpha} \leq \phi-\phi^{*} \leq 0, \quad x \in \mathbb{R}^{N-1} \\
\lim _{l \rightarrow+\infty} \sup _{\operatorname{dist}\left(x, E_{\infty}\right) \geq l}\left|\phi(x)-\phi^{*}(x)\right|=0
\end{array}\right.
$$

where $E_{\infty}$ is the set of edges defined as

$$
E_{\infty}=\left\{x \in \mathbb{R}^{N-1} \mid \phi_{\infty} \text { is not } C^{1} \text { at } x\right\}
$$

with the 1-homogeneous function

$$
\phi_{\infty}(x)=\inf _{\nu \in A}(-(\cot \alpha) x \cdot \nu) .
$$


In space dimension $N=3$, we obtain a more precise result by considering solutions having a finite number of gradient jumps. Those solutions are still more complex than the infimum of a finite number of affine forms. Here is the precise result.

Theorem 1.3 (Solutions with finite number of gradient jumps and $N=3$ )

Let $\alpha \in\left(0, \frac{\pi}{2}\right], c_{0}>0$ and $c=c_{0} / \sin \alpha$. Choose $\phi_{\infty}$ a 1-homogeneous viscosity solution to the eikonal Equation (5) in dimension $N=3$ with a finite number of singularities on $\mathbb{S}^{1}$. Then, there exist

(1) a $2 \pi$-periodic continuous function $\psi_{\infty}: \theta \in[0,2 \pi] \mapsto \psi_{\infty}(\theta) \in[-\cot \alpha, \cot \alpha]$ and a finite number $k \in \mathbb{N} \backslash\{0\}$ of angles $\theta_{1}<\cdots<\theta_{k}$ in $[0,2 \pi)$ such that

$$
\phi_{\infty}(r \cos \theta, r \sin \theta)=r \psi_{\infty}(\theta), \quad(r, \theta) \in \mathbb{R}^{+} \times[0,2 \pi) .
$$

Moreover, for any $i \in\{1, \ldots, k\}$,

(a) Either $\forall \theta \in\left[\theta_{i}, \theta_{i+1}\right], \psi_{\infty}(\theta)=-(\cot \alpha)$ and we set $\sigma_{i}=1$.

(b) $\mathrm{Or}$

$$
\left\{\begin{array}{l}
\forall \theta \in\left[\theta_{i}, \frac{\theta_{i}+\theta_{i+1}}{2}\right], \quad \psi_{\infty}(\theta)=-(\cot \alpha) \cos \left(\theta-\theta_{i}\right) \\
\forall \theta \in\left[\frac{\theta_{i}+\theta_{i+1}}{2}, \theta_{i+1}\right], \quad \psi_{\infty}(\theta)=-(\cot \alpha) \cos \left(\theta-\theta_{i+1}\right)
\end{array} \text { and we set } \sigma_{i}=0 .\right.
$$

By convention, $\theta_{k+1}=2 \pi+\theta_{1}$ and $\sigma_{k+1}=\sigma_{1}$. If $k \geq 2$, then $\sigma_{i} \sigma_{i+1}=0$ for any $i \in\{1, \ldots, k\}$.

(2) a smooth concave function $\phi \in C^{\infty}\left(\mathbb{R}^{2}\right)$ solution to Equation (3) such that when $|x|$ goes to infinity

$$
\phi(x)=\phi_{*}(x)+O(1)
$$

where

$$
\phi_{*}(x)=-\frac{2}{c_{0} \sin \alpha} \ln \left(\int_{\mathbb{S}^{1}} e^{\frac{c_{0} \cos \alpha}{2} x \cdot \nu} \mathrm{d} \mu(\nu)\right)
$$

and $\mu$ is the non negative measure on $\mathbb{S}^{1}$ with finite mass determined by $\psi_{\infty}$ as follows: We set $\mu=\sum_{i=1}^{k} \mu_{i}$ where for any fixed $\lambda_{0}>0$, we set
(a) If $\sigma_{i}=1$, then $\mu_{i}=\mathbb{1}_{\left(\theta_{i}, \theta_{i+1}\right)} \mathrm{d} \theta+\lambda_{0}\left(\delta_{\theta_{i}}+\delta_{\theta_{i+1}}\right)$ (with the exception for $k=1$ : $\mu_{1}=\mathbb{1}_{\left(\theta_{1}, \theta_{1}+2 \pi\right)} \mathrm{d} \theta$ ).
(b) If $\sigma_{i}=0$, then $\mu_{i}=\lambda_{0}\left(\delta_{\theta_{i}}+\delta_{\theta_{i+1}}\right)$.

We plan to use our travelling graphs for the forced mean curvature motion exhibited in Theorems 1.1 to 1.3 in order to construct multi-dimensional travelling fronts to the reaction diffusion Equation (4); we plan to do it in a forthcoming paper.

That Equation (5) prescribes the asymptotic behaviour of (3) has nothing surprising: let $\varepsilon>0$ and denote by $\phi_{\varepsilon}$ the scaled function

$$
\phi_{\varepsilon}(x)=\varepsilon \phi\left(\frac{x}{\varepsilon}\right), \quad x \in \mathbb{R}^{N-1} .
$$

Since $\phi$ is a solution to (3), $\phi_{\varepsilon}$ satisfies

$$
-\varepsilon \operatorname{div}\left(\frac{D \phi_{\varepsilon}}{\sqrt{1+\left|D \phi_{\varepsilon}\right|^{2}}}\right)+c_{0}-\frac{c}{\sqrt{1+\left|D \phi_{\varepsilon}\right|^{2}}}=0, \quad x \in \mathbb{R}^{N-1} .
$$

Let $\varepsilon$ go to zero. If adequate estimates for $\phi_{\varepsilon}$ are known, (a subsequence of) $\left(\phi_{\varepsilon}\right)_{\varepsilon>0}$ converges to a function $\phi_{\infty}$ satisfying (5). 
The proof of Theorem 1.1 is done by a sub and super solutions argument. We first construct a family of smooth sub-solutions to (3), which will give us some better insight in the equation. This step is quite general, and works in any space dimension. Then, we will construct a Lipschitz super-solution whose rescaled asymptotics is prescribed by $\phi_{\infty}$ and this will give us a smooth solution whose asymptotic behavior is not well precise. To get a better asymptotics of the super-solution prescribed by the sub-solution, this will require a more delicate matching procedure which will limit us, for the moment, to any space dimension $N$ with a finite number of facets (Theorem 1.2) or to the space dimension $N=3$ and a finite number of gradient jumps (Theorem 1.3).

The rest of this paper is organized as follows. In Section 2, we build and characterize all 1-homogeneous solutions to the eikonal Equation (5). In Section 3, we detail Perron's method in our context, and explain why it will yield a smooth concave solution. Subsolutions are built in Section 4, and super-solutions in Section 5. Finally, Section 6 sums up previous constructions to prove Theorems 1.1 and 1.2. Section 7 presents a more precise approach in dimension $N=3$ and details the proof of Theorem 1.3. An appendix is devoted to the Laplace's method that we use in our estimates.

\section{Acknowledgments}

The first author was partly supported by the ANR project MICA, the second and third ones by the ANR project PREFERED. They acknowledge a fruitful discussion with G. Barles and thank C. Imbert for enlightening discussions on his paper [9]. They are also indebted to H. Berestycki and CAMS Center of EHESS in Paris for their hospitality while preparing this work. Finally, they thank the referees for their work.

\section{Eikonal equation}

In this section, we classify the continuous viscosity solutions to the eikonal equation in any dimension $N \geq 2$ :

$$
\left|D \phi_{\infty}(x)\right|=\cot \alpha, \quad x \in \mathbb{R}^{N-1}
$$

where $\alpha \in\left(0, \frac{\pi}{2}\right]$ is some given angle. In a first subsection, we are interested in the general case. In a second one, we reduce our study to 1-homogeneous functions and give a better description of those solutions in order to use them in both Sections 4 and 5 .

\subsection{Characterization of solutions to (10) in any dimension $N$}

For any unit vector $\nu \in \mathbb{S}^{N-2}$ and $\gamma \in(-\infty,+\infty]$, let us define the affine map

$$
\phi_{\nu, \gamma}(x)=-(\cot \alpha) \nu \cdot x+\gamma \in(-\infty,+\infty], \quad x \in \mathbb{R}^{N-1} .
$$

Proposition 2.1 (A Liouville theorem for the eikonal equation). - Let $\phi_{\infty} \in C\left(\mathbb{R}^{N-1}\right)$. Then $\phi_{\infty}$ is a viscosity solution to the eikonal equation (10) if and only if there exists a lower semi-continuous map $\gamma: \mathbb{S}^{N-2} \rightarrow(-\infty,+\infty]$ such that

$$
\phi_{\infty}(x)=\inf _{\nu \in \mathbb{S}^{N-2}} \phi_{\nu, \gamma(\nu)}(x) .
$$

Moreover $\phi_{\infty}$ is 1-homogeneous if and only if for all $\nu \in \mathbb{S}^{N-2}, \gamma(\nu) \in\{0,+\infty\}$. 
This result is most certainly known. Because we not only need the result but also an insight of the construction, we give a complete proof.

Proof of Proposition 2.1. - We first show the direct implication. Let $\phi_{\infty} \in C\left(\mathbb{R}^{N-1}\right)$ be a viscosity solution to (10). We shall prove that $\phi_{\infty}$ is $(\cot \alpha)$-Lipschitz and concave before giving its characterization as an infimum of affine maps.

Step 1: $\phi_{\infty}$ is $(\cot \alpha)$-Lipschitz. - We simply use the fact that $\phi_{\infty}$ satisfies

$$
\left|D \phi_{\infty}\right| \leq \cot \alpha \quad \text { on } \quad \mathbb{R}^{N-1} \text {. }
$$

For any $\rho>0, \varepsilon \geq 0$ and $x_{0} \in \mathbb{R}^{N-1}$, let us define

$$
\Psi(x)=\left\{\begin{array}{lll}
\phi_{\infty}\left(x_{0}\right)+\left|x-x_{0}\right| \cot \alpha+\frac{\varepsilon}{\rho-\left|x-x_{0}\right|} & \text { if } & \left|x-x_{0}\right|<\rho \\
+\infty & \text { if } & \left|x-x_{0}\right| \geq \rho
\end{array}\right.
$$

and for any $\lambda \geq 0$, we define

$$
\Psi^{\lambda}(x)=\lambda+\Psi(x)
$$

For any $\varepsilon>0$, we can check that $\Psi^{\lambda}$ is a strict supersolution where it is finite and for $x \neq x_{0}$, i.e.,

$$
\left|D \Psi^{\lambda}(x)\right|>\cot \alpha \quad \text { on } \quad B_{\rho}\left(x_{0}\right) \backslash\left\{x_{0}\right\}, \quad \text { if } \varepsilon>0 .
$$

Then we define

$$
\lambda^{*}=\inf \left\{\lambda \geq 0, \quad \Psi^{\lambda} \geq \phi_{\infty} \quad \text { on } \quad \mathbb{R}^{N-1}\right\} .
$$

Assume that $\lambda^{*}>0$. Then this implies that there exists a point $x_{\lambda} \in B_{\rho}\left(x_{0}\right) \backslash\left\{x_{0}\right\}$ such that

$$
\begin{cases}\Psi^{\lambda^{*}} \geq \phi_{\infty} & \text { on } \quad \mathbb{R}^{N-1} \\ \Psi^{\lambda^{*}}=\phi_{\infty} & \text { at } x=x_{\lambda}\end{cases}
$$

Because $\Psi^{\lambda^{*}}$ is a test function from above for $\phi_{\infty}$, we get a contradiction between the subsolution inequality (12) and the strict supersolution inequality (13) for $\varepsilon>0$. Therefore $\lambda^{*}=0$, i.e., for $x \in B_{\rho}\left(x_{0}\right)$ :

$$
\phi_{\infty}(x)-\phi_{\infty}\left(x_{0}\right) \leq\left|x-x_{0}\right| \cot \alpha+\frac{\varepsilon}{\rho-\left|x-x_{0}\right|}
$$

which gives in the limit $\varepsilon \rightarrow 0$ :

$$
\phi_{\infty}(x)-\phi_{\infty}\left(x_{0}\right) \leq\left|x-x_{0}\right| \cot \alpha \quad \text { for any } \quad x \in B_{\rho}\left(x_{0}\right)
$$

and by symmetry and because $\rho>0$ is arbitrary, we get

$$
\left|\phi_{\infty}(x)-\phi_{\infty}\left(x_{0}\right)\right| \leq\left|x-x_{0}\right| \cot \alpha \text { for all } x, x_{0} \in \mathbb{R}^{N-1} .
$$

Step 2: $\phi_{\infty}$ is concave. - Because $\phi_{\infty}$ is a Lipschitz stationary viscosity solution to the evolution equation

$$
u_{t}+H(D u)=0, \quad x \in \mathbb{R}^{N-1} \text { where } H(p)=\frac{1}{2}\left(p^{2}-\cot ^{2} \alpha\right), \quad p \in \mathbb{R}^{N-1}
$$

we can apply Lemma 4 page 131 in [4], and get that $\phi_{\infty}$ satisfies for any $t>0$

$$
\phi_{\infty}\left(x+x^{\prime}\right)-2 \phi_{\infty}(x)+\phi_{\infty}\left(x-x^{\prime}\right) \leq C_{0} \frac{\left|x^{\prime}\right|^{2}}{t}, \quad \text { for all }\left(x, x^{\prime}\right) \in \mathbb{R}^{2(N-1)}
$$


and we can check that we have $C_{0}=1$. Letting $t$ go to infinity shows that $\phi_{\infty}$ is concave in $\mathbb{R}^{N-1}$.

Step 3: Tangent cone. - Since $\phi_{\infty}$ is Lipschitz continuous, it is differentiable almost everywhere by Rademacher's theorem. Let $D \subset \mathbb{R}^{N-1}$ be the set of differentiability of $\phi_{\infty}$ and fix $x_{0} \in D$. Since $\phi_{\infty}$ is concave, for any $x \in \mathbb{R}^{N-1}$, we have

$$
\phi_{\infty}(x) \leq \phi_{\infty}\left(x_{0}\right)+D \phi_{\infty}\left(x_{0}\right) \cdot\left(x-x_{0}\right) .
$$

Passing to the infimum on $D$, we get for any $x \in \mathbb{R}^{N-1}$,

$$
\phi_{\infty}(x) \leq \psi(x):=\inf _{x_{0} \in D} \phi_{\infty}\left(x_{0}\right)+D \phi_{\infty}\left(x_{0}\right) \cdot\left(x-x_{0}\right) .
$$

Thus, $\psi$ and $\phi_{\infty}$ are $(\cot \alpha)$-Lipschitz functions that coincide on $D$ which is a dense set on $\mathbb{R}^{N-1}$. Therefore, they are in fact equal on $\mathbb{R}^{N-1}$. Using Equation (10), we finally have

$$
\phi_{\infty}(x)=\inf _{x_{0} \in D}-(\cot \alpha) \nu\left(x_{0}\right) \cdot x+g\left(x_{0}\right)
$$

where for any $x_{0} \in D, \quad \nu\left(x_{0}\right)=-D \phi_{\infty}\left(x_{0}\right) / \cot \alpha \in \mathbb{S}^{N-2} \quad$ and $\quad g\left(x_{0}\right)=\phi_{\infty}\left(x_{0}\right)$ $-x_{0} \cdot D \phi_{\infty}\left(x_{0}\right) \in \mathbb{R}$. Defining $\gamma$ as

$$
\begin{aligned}
\gamma: \mathbb{S}^{N-2} & \rightarrow(-\infty,+\infty] \\
\nu & \mapsto\left\{\begin{array}{l}
\inf _{x_{0} \in A} g\left(x_{0}\right) \text { if } A:=\left\{x_{0} \in D \mid \nu\left(x_{0}\right)=\nu\right\} \neq \varnothing \\
+\infty \text { otherwise }
\end{array}\right.
\end{aligned}
$$

we get the desired characterization (11). Since $\phi_{\infty}$ is continuous, we also deduce from (11) that $\gamma$ is lower semi-continuous.

Step 4: The 1-homogeneous case. - We assume that $\phi_{\infty}$ is a 1-homogeneous continuous viscosity solution to (10). Then for any $x_{0} \in \mathbb{R}^{N-1}$, there exists $p \in \mathbb{R}^{N-1}$ with $|p|=\cot \alpha$ such that by (11)

$$
\forall x \in \mathbb{R}^{N-1}, \quad \phi_{\infty}(x) \leq \phi_{\infty}\left(x_{0}\right)+p \cdot\left(x-x_{0}\right) .
$$

On the one hand, considering $x=0$, we get

$$
p \cdot x_{0} \leq \phi_{\infty}\left(x_{0}\right)
$$

because $\phi_{\infty}$ is 1-homogeneous. On the other hand considering $\lambda x$ instead of $x$ and taking the limit $\lambda \rightarrow+\infty$, we get

$$
\psi(x):=p \cdot x \geq \phi_{\infty}(x) \quad \text { with equality at } \quad x=x_{0} .
$$

Therefore if we call $\mathscr{L}_{\phi_{\infty}}$ the set of linear functions $\psi$ satisfying $\psi \geq \phi_{\infty}$ such that $|\nabla \psi|=\cot \alpha$, we have

$$
\phi_{\infty}=\inf _{\psi \in \mathscr{L}_{\phi \infty}} \psi
$$

because this is true at any point $x_{0} \in \mathbb{R}^{N-1}$.

Step 5: Conclusion. - Conversely, if a function $\phi_{\infty}$ is given by (11), then it is straightforward to check that $\phi_{\infty}$ is a viscosity solution to (10). 
Remark 2.2. - In dimension $N=2$, the previous proposition simply reads: If $N=2$ and $\alpha \in\left(0, \frac{\pi}{2}\right], \phi_{\infty}$ is a viscosity solution to (10) if and only if $\phi_{\infty}$ is affine or if there exists $\left(x_{0}, y_{0}\right) \in \mathbb{R}^{2}$ such that

$$
\phi_{\infty}(x)=-(\cot \alpha)\left|x-x_{0}\right|+y_{0}, \quad x \in \mathbb{R} .
$$

Moreover, $\phi_{\infty}$ is 1-homogeneous if and only if $y_{0}=0$.

The proof of this proposition can also be done directly from definitions of viscosity solutions and we omit the details. Notice however the link with [8]: two-dimensional reaction diffusion waves are either planar fronts or the unique (up to translations) conical front whose level sets are asymptotics to the graph of $\phi_{\infty}$ just described.

\subsection{The 1-homogeneous case}

As stressed in Theorem 1.1, we only build solutions to the forced mean curvature motion Equation (3) whose asymptotics is prescribed by a 1-homogeneous solution to the eikonal Equation (10). Therefore, it is worth emphasizing this particular case.

Notice however that there exist viscosity solutions to the eikonal Equation (10) defined in $\mathbb{R}^{N-1}$ that are not homogeneous of order 1 . For instance, consider solutions given by (15) with $x \in \mathbb{R}^{N-1}$ and $y_{0} \neq 0$. We can also consider any translation of a 1-homogeneous solution. Another example is for instance given in dimension $N=3$ by a function $\phi_{\infty}=\inf _{i=1 \ldots 4} \phi_{i}$ where $\left(\phi_{i}\right)_{i \in\{1 \ldots 4\}}$ are four planar solutions defined for $x=\left(x_{1}, x_{2}\right) \in \mathbb{R}^{2}$ by

$$
\begin{array}{ll}
\phi_{1}(x)=-(\cot \alpha) x_{1}+2 & \phi_{2}(x)=(\cot \alpha) x_{1}+2 \\
\phi_{3}(x)=-(\cot \alpha) x_{2} & \phi_{4}(x)=(\cot \alpha) x_{2} .
\end{array}
$$

It is straightforward to check that $\phi_{\infty}$ satisfies $\left|D \phi_{\infty}\right|=\cot \alpha$ in the viscosity sense and that it is not homogeneous of order 1 since there exists $\lambda>0$ such that $\phi_{\infty}(\lambda, 0) \neq \lambda \phi_{\infty}(1,0)$.

In any case, a solution $\phi_{\infty}$ to the eikonal Equation (10) is concave (see the proof of Proposition 2.1, step 2). Therefore the function $g: \lambda \in \mathbb{R}^{+*} \mapsto g(\lambda)=\phi_{\infty}(\lambda x) /(\lambda|x|) \in \mathbb{R}$ is decreasing in $\lambda>0$. Since $\phi_{\infty}$ is $(\cot \alpha)$-Lipschitz, $g$ is bounded from below and for any $x \in \mathbb{S}^{N-2}$, the limit

$$
\lim _{\lambda \rightarrow+\infty} \frac{\phi_{\infty}(\lambda x)}{|\lambda|}
$$

exists and $\phi_{\infty}$ is asymptotically homogeneous. Thus we have a fairly general understanding of what is going on by restricting ourselves to homogeneous solutions to Equation (10).

Proposition 2.3 (A countable characterization of homogeneous solutions)

Let $\phi_{\infty} \in C\left(\mathbb{R}^{N-1}\right)$. Then $\phi_{\infty}$ is a 1-homogeneous viscosity solution to the eikonal Equation (10) if and only if there exists a sequence $\left(\nu_{i}\right)_{i \in \mathbb{N}}$ of $\mathbb{S}^{N-2}$ such that

$$
\phi_{\infty}(x)=\inf _{i \in \mathbb{N}}-(\cot \alpha) \nu_{i} \cdot x
$$

Proof of Proposition 2.3. - Let $\phi_{\infty} \in C\left(\mathbb{R}^{N-1}\right)$ be a 1-homogeneous viscosity solution to (10). According to Proposition 2.1, there exists a lower semi continuous function $\gamma$ defined from $\mathbb{S}^{N-2}$ to $\{0,+\infty\}$ such that

$$
\phi_{\infty}(x)=\inf _{\nu \in \mathbb{S}^{N-2}} \phi_{\nu, \gamma(\nu)}(x), \quad x \in \mathbb{R}^{N-1} .
$$

4 e SÉRIE - TOME $46-2013$ - No 2 
Then $K=\left\{\nu \in \mathbb{S}^{N-2} \mid \gamma(\nu)=0\right\}$ is a compact set of $\mathbb{S}^{N-2}$. We claim (see Lemma 2.4 and Corollary 2.5 below) that there exists a sequence $\left(\nu_{i}\right)_{i \in \mathbb{N}}$ of $\mathbb{S}^{N-2}$ such that

$$
K=\overline{\bigcup_{i \in \mathbb{N}}\left\{\nu_{i}\right\}}
$$

Thus, $\phi_{\infty}(x)$ can be described as the infimum over $\nu \in K$ of the linear functions $-(\cot \alpha) x \cdot \nu$. Since $\cup_{i \in \mathbb{N}}\left\{\nu_{i}\right\}$ is dense in $K, \phi_{\infty}(x)$ can also be written as the infimum over $i \in \mathbb{N}$ of the linear functions $-(\cot \alpha) x \cdot \nu_{i}$. This ends the proof of Proposition 2.3 since the converse implication is straightforward.

Lemma 2.4 (Decomposition of a compact set of $\mathbb{S}^{N-2}$ in cubes). - For any compact set $K$ of $\mathbb{S}^{N-2}$, there exists a countable family $\left(Q_{i}\right)_{i \in \mathbb{N}}$ of closed cubes of $\mathbb{R}^{N-1}$ such that

$$
\left\{\begin{array}{l}
\forall n \in \mathbb{N}, \quad K \subset \bigcup_{i \geq n} Q_{i} \\
\forall i \in \mathbb{N}, \quad Q_{i} \cap K \neq \varnothing \\
\limsup _{i \rightarrow+\infty} \operatorname{diam}\left(Q_{i}\right)=0 .
\end{array}\right.
$$

Proof of Lemma 2.4. - We built this decomposition into cubes by induction. Let $C_{0}=[-1,1]^{N-1}$ be the first cube of width 2 . Thus $K \subset C_{0}$. We divide $C_{0}$ in $2^{N-1}$ smaller cubes of width $2^{0}=1$. Since $C_{0} \cap K$ is not empty, there exist some of the smaller cubes called $C_{1, i}$ for $i=1 \ldots n_{1}$ whose intersection with $K$ is not empty. Then, $1 \leq n_{1} \leq 2^{N-1}$ and

$$
K \subset \bigcup_{i=1}^{n_{1}} C_{1, i} .
$$

In the same way, for $i=1, \ldots, n_{1}$, we divide each cube $C_{1, i}$ into $2^{N-1}$ smaller cubes of width $2^{-1}$ and keep only those whose intersection with $K$ is not empty. We call them $C_{2, k}$ for $k=1 \ldots n_{2}$ and $1 \leq n_{2} \leq 2^{N-1} n_{1}$. Then, one can easily verify that $K \subset \cup_{k=1 \ldots n_{2}} C_{2, k}$.

Assume the cubes $C_{j, i}$ are built for $j \in \mathbb{N}, i=1 \ldots n_{j}$ and $1 \leq n_{j} \leq 2^{j(N-1)}$ such that

$$
\left\{\begin{array}{l}
K \subset \bigcup_{i=1}^{n_{j}} C_{j, i} \\
\forall i=1 \ldots n_{j}, \quad C_{j, i} \cap K \neq \varnothing \\
\operatorname{diam}\left(C_{j, i}\right)=2^{-j+1} .
\end{array}\right.
$$

Then we construct the cubes $C_{j+1, i}$ as follows. We divide each cube $C_{j, i}$ into $2^{N-1}$ smaller cubes of width $2^{-j}$ and keep only those whose intersection with $K$ is not empty. We call them $C_{j+1, i}$ for $i=1 \ldots n_{j+1}$ and $1 \leq n_{j+1} \leq 2^{N-1} n_{j} \leq 2^{(j+1)(N-1)}$. By construction, it is easy to verify that

$$
\left\{\begin{array}{l}
K \subset \bigcup_{i=1}^{n_{j+1}} C_{j+1, i} \\
\forall i=1 \ldots n_{j+1}, \quad C_{j+1, i} \cap K \neq \varnothing \\
\operatorname{diam}\left(C_{j+1, i}\right)=2^{-j} .
\end{array}\right.
$$

The induction is then proved. We thus construct a countable family of cubes that we recall $\left(Q_{j}\right)_{j \in \mathbb{N}}$ for convenience with the desired assumptions (17). This ends the proof of Lemma 2.4. 
Corollary 2.5 (Representation of a compact set of $\mathbb{S}^{N-2}$ ). - For any compact set $K$ of $\mathbb{S}^{N-2}$, there exists a sequence $\left(\nu_{j}\right)_{j \in \mathbb{N}}$ of $\mathbb{S}^{N-2}$ such that

$$
K=\overline{\bigcup_{j \in \mathbb{N}}\left\{\nu_{j}\right\}} \text {. }
$$

Proof of Corollary 2.5. - For $K$ a compact set of $\mathbb{S}^{N-2}$, we define $\left(Q_{j}\right)_{j \in \mathbb{N}}$ a family of cubes as proposed in Lemma 2.4. For any $j \in \mathbb{N}$, we choose $\nu_{j} \in K \cap Q_{j}$. Then, it is straightforward to check that $\overline{\cup_{j \in \mathbb{N}}\left\{\nu_{j}\right\}} \subset K$. Regarding the converse inclusion, we fix $x_{0} \in K$ and $\varepsilon>0$. By (17), there exists $n_{\varepsilon} \in \mathbb{N}$ such that the width of cube $Q_{i}$ is smaller than $\varepsilon$ provided $i \geq n_{\varepsilon}$. Since $K \subset \cup_{i \geq n_{\varepsilon}} Q_{i}$, there exists $i_{\varepsilon} \geq n_{\varepsilon}$ such that

$$
x_{0} \in Q_{i_{\varepsilon}} \text { and }\left|x_{0}-\nu_{i_{\varepsilon}}\right| \leq \varepsilon \sqrt{N-1} .
$$

This shows the density of $\cup_{j \in \mathbb{N}}\left\{\nu_{j}\right\}$ in $K$ and ends the proof of Corollary 2.5.

\section{Perron's method and comparison principle}

In this section, we are concerned with the forced mean curvature motion equation

$$
-\operatorname{div}\left(\frac{D \phi}{\sqrt{1+|D \phi|^{2}}}\right)+c_{0}-\frac{c}{\sqrt{1+|D \phi|^{2}}}=0, \quad x \in \mathbb{R}^{N-1}
$$

with the condition at infinity

$$
\phi(x)=\phi_{\infty}(x)+o(|x|), \quad x \in \mathbb{R}^{N-1}
$$

where $\phi_{\infty}$ is a homogeneous viscosity solution to $\left|D \phi_{\infty}\right|=\cot \alpha$ found in Section 2 with $\alpha=\arcsin \left(c_{0} / c\right) \in\left(0, \frac{\pi}{2}\right]$. We choose to solve (18) using Perron's method with sub and super-solutions (see [3] or [6]). Let us first recall the existence process and clarify the regularity of the solution in the following

Proposition 3.1 (Existence of a solution to (18) in dimension $N$ ). - Let $N \in \mathbb{N} \backslash\{0,1\}$, $\left(c_{0}, c\right) \in \mathbb{R}^{2}$ such that $c \geq c_{0}>0$. Assume that $\phi_{*}$ is a viscosity sub-solution and $\phi^{*}$ a viscosity super-solution to (18) such that $\phi_{*} \leq \phi^{*}$ on $\mathbb{R}^{N-1}$. Then,

(i) there exists a function $\phi \in\left[\phi_{*}, \phi^{*}\right]$ viscosity solution to (18).

(ii) Moreover, if $\phi^{*}$ is concave, and satisfies the following technical condition:

$$
\text { there exists } p \in \mathbb{R}^{N-1} \text { such that } \limsup _{|x| \rightarrow+\infty} \frac{\phi^{*}(x)-p \cdot x}{|x|}<0 \text {, }
$$

then $\phi$ can be chosen concave and smooth.

Proof of Proposition 3.1. - We build the solution $\phi$ using Perron's method directly in the framework of viscosity solutions to (18), that is to say $\phi$ is chosen as the maximal subsolution to (18) (see the user's guide to viscosity solutions [3]).

Step 1: Concavity. - We apply a result due to Imbert (see [9]) that we first recall. Denote $F$ the following Hamiltonian

$F(p, M)=-\frac{\operatorname{tr} M}{\sqrt{1+|p|^{2}}}+\frac{\operatorname{tr}(M \cdot(p \otimes p))}{\left(1+|p|^{2}\right)^{3 / 2}}+c_{0}-\frac{c}{\sqrt{1+|p|^{2}}}, \quad(p, M) \in \mathbb{R}^{N-1} \times \mathbb{R}_{\mathrm{sym}}^{(N-1) \times(N-1)}$

where $\mathbb{R}_{\text {sym }}^{(N-1) \times(N-1)}$ is the set of $(N-1)$-square symmetric matrices.

$4^{\text {e }}$ SÉRIE - TOME $46-2013-$ No $^{\circ} 2$ 
Proposition 3.2 (Imbert's Proposition 5 in [9]). - Let u be a lower semi-continuous and epipointed function. If $u$ is a supersolution to

$$
F\left(D u(x), D^{2} u(x)\right)=0, \quad x \in \mathbb{R}^{N-1}
$$

then, so is its convex envelope.

In our context, $-\phi^{*}$ is epi-pointed because of the technical condition (20). Thus, the maximal sub-solution $\phi$ to (18) is concave (otherwise Imbert's result stated in Proposition 3.2 contradicts the maximal property of $\phi$ ). Then, $\phi$ is a concave viscosity solution to (18).

Step 2: Regularity. - Once concavity is at hand, a Lipschitz bound is automatically available from the Equation (18) itself:

$$
|D \phi(x)| \leq \cot \alpha, \quad x \in \mathbb{R}^{N-1}
$$

where $\alpha \in\left(0, \frac{\pi}{2}\right]$ is such that $c_{0}=c \sin \alpha$. Then $F$ becomes uniformly elliptic, thus allowing for $C^{1,1}$ estimates (see Theorem 4 in [9]). A bootstrap argument then shows that the solution is $C^{\infty}$. This concludes the proof of Proposition 3.1.

Remark 3.3. - Notice that the condition (20) is hidden in the statement of Proposition 9 in [9]. Thus, the proof of Proposition 5 in [9] uses Proposition 9.

It now remains to find sub and super-solutions to (18).

\section{Sub-solution}

In this section we build smooth sub-solutions to the forced mean curvature Equation (18) as global solutions to a viscous eikonal equation and we do believe that they are really close to the desired solutions.

\subsection{Sub-solutions as solutions to a viscous eikonal equation}

We have the following

Lemma 4.1 (Sufficient condition for a sub-solution to (18)). - Fix $\alpha \in\left(0, \frac{\pi}{2}\right], c_{0}>0$ and $c=c_{0} / \sin \alpha$. Let $\phi_{*}$ be a concave smooth solution to

$$
-\Delta \phi_{*}=\frac{c_{0} \sin \alpha}{2}\left(\cot ^{2} \alpha-\left|D \phi_{*}\right|^{2}\right), \quad x \in \mathbb{R}^{N-1}
$$

such that

$$
\left|D \phi_{*}(x)\right| \leq \cot \alpha, \quad x \in \mathbb{R}^{N-1} .
$$

Then $\phi_{*}$ is a smooth sub-solution to Equation (18). 
Proof of Lemma 4.1. - Let $\phi_{*}$ be any concave function verifying (21) and (22). Since $\phi_{*}$ is smooth and concave, we have

$$
\begin{aligned}
N\left[\phi_{*}\right]:= & -\operatorname{div}\left(\frac{D \phi_{*}}{\sqrt{1+\left|D \phi_{*}\right|^{2}}}\right)+c_{0}-\frac{c}{\sqrt{1+\left|D \phi_{*}\right|^{2}}} \\
& =-\frac{\Delta \phi_{*}}{\sqrt{1+\left|D \phi_{*}\right|^{2}}}+\frac{D^{2} \phi_{*}\left(D \phi_{*}, D \phi_{*}\right)}{\left(1+\left|D \phi_{*}\right|^{2}\right)^{\frac{3}{2}}}+c_{0}-\frac{c}{\sqrt{1+\left|D \phi_{*}\right|^{2}}} \\
& \leq \frac{1}{\sqrt{1+\left|D \phi_{*}\right|^{2}}}\left(-\Delta \phi_{*}+c_{0} \sqrt{1+\left|D \phi_{*}\right|^{2}}-c\right) .
\end{aligned}
$$

From (22) and $c_{0}=c \sin \alpha$, we deduce that

$$
\begin{aligned}
\cot ^{2} \alpha-\left|D \phi_{*}\right|^{2} & =\left(\frac{c}{c_{0}}\right)^{2}-\left(\sqrt{1+\left|D \phi_{*}\right|^{2}}\right)^{2} \\
& \leq \frac{2 c}{c_{0}}\left(\frac{c}{c_{0}}-\sqrt{1+\left|D \phi_{*}\right|^{2}}\right) .
\end{aligned}
$$

Using Equation (21) satisfied by $\phi_{*}$, we get

$$
N\left[\phi_{*}\right] \leq \frac{1}{\sqrt{1+\left|D \phi_{*}\right|^{2}}}\left(\frac{c}{c_{0}}-\sqrt{1+\left|D \phi_{*}\right|^{2}}\right)\left(\frac{2 c}{c_{0}} \frac{c_{0}^{2}}{2 c}-c_{0}\right)=0 .
$$

Thus, $\phi_{*}$ is a sub-solution to (18).

As it is well-known, Equation (21) is readily transformed into a linear one by the HopfCole transform

$$
\tilde{\phi}_{*}(x)=\exp \left(-\frac{c_{0} \sin \alpha}{2} \phi_{*}\left(\frac{2 x}{c_{0} \cos \alpha}\right)\right), \quad x \in \mathbb{R}^{N-1}, \quad \alpha \neq \frac{\pi}{2}
$$

where $\tilde{\phi}_{*}$ is a positive solution to

$$
-\Delta \tilde{\phi}_{*}(x)+\tilde{\phi}_{*}(x)=0, \quad x \in \mathbb{R}^{N-1} .
$$

From [2], a positive solution $\tilde{\phi}_{*}$ to (23) has the form

$$
\tilde{\phi}_{*}(x)=\int_{\mathbb{S}^{N-2}} e^{\nu \cdot x} \mathrm{~d} \mu(\nu), \quad x \in \mathbb{R}^{N-1},
$$

where $\mu$ is a non negative measure on $\mathbb{S}^{N-2}$ with finite mass.

Now, for any non negative measure $\mu$ on the sphere $\mathbb{S}^{N-2}$, let us define

$$
\phi_{*}(x)=-\frac{2}{c_{0} \sin \alpha} \ln \left(\int_{\mathbb{S}^{N-2}} e^{\frac{c_{0} \cos \alpha}{2} x \cdot \nu} \mathrm{d} \mu(\nu)\right), \quad x \in \mathbb{R}^{N-1}, \quad \alpha \in\left(0, \frac{\pi}{2}\right] .
$$

By construction, $\phi_{*}$ is a smooth solution to (21). Let us now prove that $\phi_{*}$ is a sub-solution to Equation (18), with all the requirements.

Lemma 4.2 (Inequalities for the derivatives of $\phi_{*}$ ). - Let $\mu$ be a non negative measure on $\mathbb{S}^{N-2}$ with finite mass, $\alpha \in\left(0, \frac{\pi}{2}\right], c_{0}>0$ and $c=c_{0} / \sin \alpha$. Define $\phi_{*}$ as in (24). Then $\phi_{*}$ is a smooth concave solution to (21) and its gradient is uniformly bounded, that is to say, for any $(x, \xi) \in \mathbb{R}^{N-1} \times \mathbb{R}^{N-1}$,

$$
\left|D \phi_{*}(x)\right| \leq \cot \alpha, \quad D^{2} \phi_{*}(x)(\xi, \xi) \leq 0 .
$$


Proof of Lemma 4.2. - Let $\mu$ and $\phi_{*}$ be so defined. We have

$$
D \phi_{*}(x)=-(\cot \alpha) \frac{F_{\nu}(x)}{F_{1}(x)}, \quad x \in \mathbb{R}^{N-1}
$$

where for any continuous (scalar or vector) function $f$ defined on $\mathbb{S}^{N-2}$

$$
F_{f}(x)=\int_{\mathbb{S}^{N-2}} e^{\frac{c_{0} \cos \alpha}{2} x \cdot \nu} f(\nu) \mathrm{d} \mu(\nu) .
$$

Remark that if we define for some fixed $x \in \mathbb{R}^{N-1}, \int_{\mathbb{S}^{N-2}} f(\nu) \mathrm{d} \bar{\mu}_{x}(\nu)=\frac{F_{f}(x)}{F_{1}(x)}$, then $\bar{\mu}_{x}$ is a probability measure on $\mathbb{S}^{N-2}$. We can then apply Jensen's inequality to the convex function $y \mapsto|y|^{2}$. This gives

$$
\left|\frac{F_{f}(x)}{F_{1}(x)}\right|^{2}=\left|\int_{\mathbb{S}^{N-2}} f(\nu) \mathrm{d} \bar{\mu}_{x}\right|^{2} \leq \int_{\mathbb{S}^{N-2}}|f(\nu)|^{2} \mathrm{~d} \bar{\mu}_{x}=\frac{F_{|f|^{2}}(x)}{F_{1}(x)}
$$

for any continuous function $f$ defined on $\mathbb{S}^{N-2}$. Applying this inequality to $f(\nu)=\nu$, we get the desired bound on the gradient of $\phi_{*}:\left|D \phi_{*}(x)\right| \leq \cot \alpha$.

Regarding the concavity property of $\phi_{*}$, we use the same type of arguments. Indeed, for any $\xi \in \mathbb{R}^{N-1}$ and $x \in \mathbb{R}^{N-1}$, we have

$$
D^{2} \phi_{*}(x)(\xi, \xi)=-\frac{c_{0} \cos ^{2} \alpha}{2 \sin \alpha}\left(\frac{F_{f^{2}}(x)}{F_{1}(x)}-\left(\frac{F_{f}(x)}{F_{1}(x)}\right)^{2}\right)
$$

where $f$ is the continuous function defined on $\mathbb{S}^{N-2}$ by $f(\nu)=\nu \cdot \xi$. Applying again Jensen's inequality (26), we conclude that $D^{2} \phi_{*}(x)(\xi, \xi) \leq 0$ for any $\xi \in \mathbb{R}^{N-1}$ and $x \in \mathbb{R}^{N-1}$ which shows that $\phi_{*}$ is concave.

Finally, we proved the following proposition:

Proposition 4.3 (Existence of a sub-solution to (18)). - Fix $\alpha \in\left(0, \frac{\pi}{2}\right], c_{0}>0$ and $c=c_{0} / \sin \alpha$. Let $\mu$ be a non negative measure on $\mathbb{S}^{N-2}$ with finite mass. Define $\phi_{*}$ as in (24). Then, $\phi_{*}$ is a smooth concave sub-solution to (18).

Remark 4.4. - The way we choose the measure $\mu$ is decisive in the asymptotic behavior of the sub-solution $\phi_{*}$ built as in Proposition 4.3. Indeed, if we want the subsolution (and hence the solution) to the mean curvature Equation (18) to follow asymptotically some given solution $\phi_{\infty}$ to the eikonal Equation (10), we will have to choose the measure $\mu$ carefully. In that procedure, information collected in Section 2 will help.

Of course, it will be also very interesting to assess whether each sub-solution built with a general probability measure gives rise to a solution to the mean curvature Equation (18).

\section{Super-solution}

A natural super-solution to the forced mean curvature Equation (18) is a viscosity solution $\phi_{\infty}$ to the eikonal Equation (10). Indeed, $\phi_{\infty}$ satisfies (in the distributional and viscosity sense)

$$
-\operatorname{div}\left(\frac{D \phi_{\infty}}{\sqrt{1+\left|D \phi_{\infty}\right|^{2}}}\right)+c_{0}-\frac{c}{\sqrt{1+\left|D \phi_{\infty}\right|^{2}}} \geq 0, \quad x \in \mathbb{R}^{N-1}
$$


However, this super-solution does not satisfy the right comparison with the previous subsolution $\phi_{*}$. For instance, if $N=3, \phi_{\infty}$ is the radially symmetric viscosity solution to the eikonal Equation (10) and $\phi_{*}$ the sub-solution associated with the Lebesgue measure $\mu=\mathrm{d} \theta$ on $[0,2 \pi]$ as in (24), then we can compute the asymptotic behavior of both functions $\phi_{\infty}$ and $\phi_{*}$ for $x \in \mathbb{R}^{2}$ with $|x|$ large enough using Laplace's method (see Appendix 8). We then observe that the sub-solution $\phi_{*}$ is above the super-solution $\phi_{\infty}$ in this area. This contradicts the crucial assumption $\phi_{*} \leq \phi^{*}$ on $\mathbb{R}^{2}$ in the Perron's method (see Proposition 3.1).

\subsection{Super-solutions as infimum of hyperplanes}

Since we do believe that the sub-solution is close to the viscosity solution to the forced mean curvature Equation (18) at infinity, we prefer to change the super-solution. In the general case of dimension $N$, we use the countable characterization of the solution $\phi_{\infty}$ to the eikonal Equation (10) that we want to approach (see Proposition 2.3).

Proposition 5.1 (Existence of a super-solution to (18)). - Fix $\alpha \in\left(0, \frac{\pi}{2}\right], c_{0}>0$ and $c=c_{0} / \sin \alpha$. Choose $\phi_{\infty}$ a 1-homogeneous solution to the eikonal Equation (10). Define $\left(\nu_{i}\right)_{i \in \mathbb{N}}$ the sequence of $\mathbb{S}^{N-2}$ given by its countable characterization in Proposition 2.3.

For any sequence $\left(\lambda_{i}\right)_{i \in \mathbb{N}}$ such that $\lambda_{i}>0$ and $\sum_{i \in \mathbb{N}} \lambda_{i}<+\infty$, we set

$$
\phi_{i}(x)=-(\cot \alpha) x \cdot \nu_{i}-\frac{2}{c_{0} \sin \alpha} \ln \lambda_{i}, \quad i \in \mathbb{N}, \quad x \in \mathbb{R}^{N-1}
$$

and

$$
\phi^{*}(x)=\inf _{i \in \mathbb{N}} \phi_{i}(x), \quad x \in \mathbb{R}^{N-1} .
$$

Then, $\phi^{*}$ is a concave continuous super-solution to (18).

Proof of Proposition 5.1. - Since $\phi_{i}$ are exact solutions to the forced mean curvature Equation (18), it is clear that $\phi^{*}$ is a super-solution to that equation. As the infimum of affine functions, it is concave and continuous.

Remark 5.2. - This construction is very easy. However, it is not clear whether the technical condition (20) is satisfied or not. It is even clear that when the set of $\left\{\nu_{i}\right\}_{i \in \mathbb{N}}$ is finite of cardinal less or equal to $N-1$, this condition is NOT verified. We will see later (see Step 4 of the proof of Theorem 1.1) how to modify the sub-and super-solutions in order to satisfy condition (20) and then pass to the limit to recover the general case.

Remark 5.3. - In the case when the set $\left\{\nu_{i}\right\}_{i \in \mathbb{N}}$ is infinite, the convergence of $\sum \lambda_{i}$ forces $\left(\lambda_{i}\right)_{i \in \mathbb{N}}$ to go to zero and the sequence $\left(-\ln \lambda_{i}\right)_{i \in \mathbb{N}}$ grows as $i$ goes to infinity.

$4^{\mathrm{e}}$ SÉRIE - TOME $46-2013$ - No 2 


\section{General existence results}

Now equipped with sub and super-solutions as well as a Perron's method, we are able to prove existence results. The general case in dimension $N \geq 2$ is the easiest one since the asymptotics is less precise. Let us explain our ideas in details depending on the degree of precision we want to obtain in our construction.

Let $N \in \mathbb{N} \backslash\{0,1\}, \alpha \in\left(0, \frac{\pi}{2}\right], c_{0}>0$ and $c=c_{0} / \sin \alpha$. It is worth noticing that some of our constructions do not work for $\alpha=\pi / 2$. However, this case is obvious and leads to planar fronts. Therefore, we restrict ourselves to $\alpha \in\left(0, \frac{\pi}{2}\right)$.

\subsection{Proof of Theorem 1.1}

Step 1: Sub and super-solutions. - Choose $\phi_{\infty}$ a 1-homogeneous continuous viscosity solution to the eikonal Equation (10) in $\mathbb{R}^{N-1}$. By Proposition 2.3, there exists a sequence $\left(\nu_{i}\right)_{i \in \mathbb{N}}$ of $\mathbb{S}^{N-2}$ such that

$$
\phi_{\infty}(x)=\inf _{i \in \mathbb{N}}-(\cot \alpha) \nu_{i} \cdot x
$$

Let $\mu$ be the probability measure on $\mathbb{S}^{N-2}$ defined as

$$
\mu=\sum_{i \in \mathbb{N}} \lambda_{i} \delta_{\nu_{i}}
$$

where $\left(\lambda_{i}\right)_{i \in \mathbb{N}}$ are chosen so that $\lambda_{i}>0$ and $\sum_{i=0}^{+\infty} \lambda_{i}=1$.

Build the sub-solution $\phi_{*}$ as in (24) with the above measure $\mu$. Then by Proposition 4.3, $\phi_{*}$ is a smooth concave sub-solution to the mean curvature motion Equation (18). Build a concave continuous super-solution $\phi^{*}$ by Proposition 5.1 as the infimum of hyperplanes where the $\left(\lambda_{i}\right)_{i \in \mathbb{N}}$ and $\left(\nu_{i}\right)_{i \in \mathbb{N}}$ are defined by the choice of $\mu$. For any $x \in \mathbb{R}^{N-1}$,

$$
\phi_{*}(x)=-\frac{2}{c_{0} \sin \alpha} \ln \left(\sum_{i=0}^{+\infty} \lambda_{i} e^{\frac{c_{0} \cos \alpha}{2} \nu_{i} \cdot x}\right) \leq-\frac{2}{c_{0} \sin \alpha} \ln \left(\lambda_{i} e^{\frac{c_{0} \cos \alpha}{2} \nu_{i} \cdot x}\right) .
$$

Since the last inequality holds for any $i \in \mathbb{N}$, we have

$$
\phi_{*}(x) \leq \inf _{i \in \mathbb{N}}\left(-(\cot \alpha) \nu_{i} \cdot x-\frac{2}{c_{0} \sin \alpha} \ln \lambda_{i}\right)=\inf _{i \in \mathbb{N}} \phi_{i}(x)=\phi^{*}(x)
$$

and the super-solution $\phi^{*}$ is above the sub-solution $\phi_{*}$.

Step 2: Asymptotics of sub and super-solutions. - Let us now precise their asymptotics: we claim that as $|x|$ goes to infinity

$$
\phi_{*}(x)=\phi_{\infty}(x)+o(|x|) \text { and } \phi^{*}(x)=\phi_{\infty}(x)+o(|x|) .
$$

To prove such a claim, the idea is to compare $\phi_{\infty}(x)$ with the limits as $\varepsilon$ goes to zero of $\varepsilon \phi_{*}(x / \varepsilon)$ and $\varepsilon \phi^{*}(x / \varepsilon)$. In particular, we will prove the sequence of three inequalities: for any $x \in \mathbb{R}^{N-1}$

$$
\phi_{\infty}(x) \leq \lim _{\varepsilon \rightarrow 0} \varepsilon \phi_{*}(x / \varepsilon) \leq \lim _{\varepsilon \rightarrow 0} \varepsilon \phi^{*}(x / \varepsilon) \leq \phi_{\infty}(x)
$$

which proves the desired claim (27).

The first step of the present proof leads easily to the second inequality in (28) since $\phi_{*} \leq \phi^{*}$ on $\mathbb{R}^{N-1}$. As far as the first inequality is concerned, we have for any $i \in \mathbb{N}$ and $x \in \mathbb{R}^{N-1}$,

$$
\left|x \cdot \nu_{i}\right|=|x| \cos \left(\theta_{x}-\theta_{i}\right) \leq|x| \cos \delta_{x}
$$


where $\delta_{x}$ is the angular distance between $x /|x|$ and $K:=\overline{\cup_{i \in \mathbb{N}}\left\{\nu_{i}\right\}}$. Thus,

$$
\begin{aligned}
\phi_{*}(x) & \geq-\frac{2}{c_{0} \sin \alpha} \ln \left(e^{\frac{c_{0} \sin \alpha}{2}|x| \cos \delta_{x}} \mu\left(\mathbb{S}^{N-2}\right)\right) \\
& =-(\cot \alpha)|x| \cos \delta_{x}=\inf _{\nu \in K}-(\cot \alpha) x \cdot \nu=\phi_{\infty}(x)
\end{aligned}
$$

where $\mu\left(\mathbb{S}^{N-2}\right)=\sum_{i} \lambda_{i}=1$. Thus $\phi_{\infty} \leq \phi_{*}$ on $\mathbb{R}^{N-1}$ and the homogeneity of $\phi_{\infty}$ gives the first inequality of (28).

Regarding the last inequality in (28), we know that for any $x \in \mathbb{R}^{N-1}$,

$$
\phi^{*}(x) \leq \phi_{i}(x)=-(\cot \alpha) x \cdot \nu_{i}-\frac{2}{c_{0} \sin \alpha} \ln \lambda_{i} .
$$

Since $\lim _{\varepsilon \rightarrow 0} \varepsilon \phi_{i}(x / \varepsilon)=-(\cot \alpha) x \cdot \nu_{i}$, it is clear that

$$
\lim _{\varepsilon \rightarrow 0} \varepsilon \phi^{*}\left(\frac{x}{\varepsilon}\right) \leq \inf _{i \in \mathbb{N}}-(\cot \alpha) x \cdot \nu_{i}=\phi_{\infty}(x) .
$$

This ends the proof of the three inequalities (28) and hence of (27).

Step 3: Existence of a solution. - By Proposition 3.1, there exists a function $\phi \in\left[\phi_{*}, \phi^{*}\right]$ viscosity solution to (18) and by the previous step, $\phi$ verifies the right asymptotics

$$
\phi(x)=\phi_{\infty}(x)+o(|x|) .
$$

However, in the statement of Theorem 1.1, we claim that there exists a smooth concave solution to (18) and the above construction does not provide such information. By Proposition 3.1, the regularity and concavity of the solution are at hand if the super-solution $\phi^{*}$ satisfies the technical assumption (20). If it does not, we will first modify the sub and the super-solutions in order to satisfy (20), then get a concave solution, and in a last step pass to the limit to find a solution (still concave) between $\phi_{*}$ and $\phi^{*}$.

Step 4: Regularity and concavity. - Let us consider for any $\varepsilon>0$

$$
\phi_{*}^{\varepsilon}(x)=-\frac{2}{c_{0} \sin \alpha} \ln \left(\int_{\mathbb{S}^{N-2}} e^{\frac{c_{0} \cos \alpha}{2} x \cdot \nu} \mathrm{d} \mu_{\varepsilon}(\nu)\right), \quad x \in \mathbb{R}^{N-1}
$$

with $\mu_{\varepsilon}=\mu+\varepsilon \mu_{1}$ where

$$
\mu_{1}=\sum_{ \pm} \sum_{j=1}^{N-1} \delta_{ \pm e_{j}}
$$

denoting $\left(e_{i}\right)_{i \in\{1, \ldots, N-1\}}$ as the canonical orthonormal basis of $\mathbb{R}^{N-1}$. In the same way, we define

$\phi^{\varepsilon *}(x)=\inf _{i \in \mathbb{N}, j=1 \ldots N-1, \pm}\left(-(\cot \alpha) x \cdot \nu_{i}-\frac{2}{c_{0} \sin \alpha} \ln \lambda_{i},-(\cot \alpha) x \cdot\left( \pm e_{j}\right)-\frac{2}{c_{0} \sin \alpha} \ln \varepsilon\right)$.

Then, $\phi_{*}^{\varepsilon}$ is a sub-solution, $\phi^{\varepsilon *}$ is a super-solution and $\phi_{*}^{\varepsilon} \leq \phi^{\varepsilon *}$. It satisfies (20) for any $\varepsilon>0$ and for $p=0$. By Proposition 3.1, there exists a concave smooth solution $\phi^{\varepsilon}$ satisfying Equation (18), with $\phi^{\varepsilon}$ being ( $\left.\cot \alpha\right)$-Lipschitz such that

$$
\phi_{*}^{\varepsilon}(x) \leq \phi^{\varepsilon}(x) \leq \phi^{\varepsilon *}(x), \quad x \in \mathbb{R}^{N-1} .
$$

Finally, we take the limit as $\varepsilon$ goes to zero. The sub-solutions $\phi_{*}^{\varepsilon}$ go to $\phi_{*}$. The super-solutions $\phi^{\varepsilon *}$ converge to $\phi^{*}$. This follows from the expression of super-solutions as an infimum of hyperplanes, those associated to the $\varepsilon$ weights going to $+\infty$. Moreover by Ascoli's theorem, 
$\left(\phi^{\varepsilon}\right)_{\varepsilon>0}$ converges (up to a subsequence) to some concave and ( $\left.\cot \alpha\right)$-Lipschitz function $\phi^{0}$ solution to (18) and satisfying

$$
\phi_{*} \leq \phi^{0} \leq \phi^{*} .
$$

Again a bootstrap argument shows that $\phi^{0}$ is smooth. Therefore, $\phi^{0}$ is the intended solution to the mean curvature Equation (18).

\subsection{Proof of Theorem 1.2}

Step 1: Existence of a solution. - Choose $\phi^{*}$ the viscosity solution to the eikonal Equation (10) given by

$$
\phi^{*}(x)=\inf _{\nu \in A}\left(-(\cot \alpha) x \cdot \nu+\gamma_{\nu}\right), \quad x \in \mathbb{R}^{N-1}
$$

where $A=\left\{\nu_{1}, \ldots, \nu_{k}\right\}$ is a finite subset of the sphere $\mathbb{S}^{N-2}, k \in \mathbb{N}^{*}$ and $\gamma_{\nu}$ are any given real numbers. We build a sub-solution $\phi_{*}$ as in Proposition 4.3

$$
\phi_{*}(x)=-\frac{2}{c_{0} \sin \alpha} \ln \left(\sum_{i=1}^{k} \lambda_{i} e^{\frac{c_{0} \cos \alpha}{2} x \cdot \nu_{i}}\right), \quad x \in \mathbb{R}^{N-1}
$$

where $\lambda_{i}$ is determined by the relation $\gamma_{\nu_{i}}=-\frac{2}{c_{0} \sin \alpha} \ln \lambda_{i}$ for $i=1 \ldots k$. Let us notice that in the particular case when $A$ is finite, the super-solution built in Proposition 5.1 coincides with the solution $\phi^{*}$ to the eikonal equation. As in Section $6.1, \phi_{*} \leq \phi^{*}$ and the assumptions of Proposition 3.1 i) are satisfied. Thus, there exists a function $\phi \in\left[\phi_{*}, \phi^{*}\right]$ viscosity solution to (18). Dealing as in Section 6.1 step 4, we can even find a smooth concave solution still denoted $\phi \in\left[\phi_{*}, \phi^{*}\right]$. It now remains to study $\phi_{*}-\phi^{*}$ to get a precise asymptotics of the solution $\phi$.

Step 2: Asymptotics (first line of (8)). - Setting

$$
\phi_{i}(x)=-(\cot \alpha) x \cdot \nu_{i}-\frac{2}{c_{0} \sin \alpha} \ln \lambda_{i}
$$

we have

$$
\begin{aligned}
\phi_{*}(x) & =-\frac{2}{c_{0} \sin \alpha} \ln \left(\sum_{i=1}^{k} e^{-\frac{c_{0} \sin \alpha}{2} \phi_{i}(x)}\right) \geq-\frac{2}{c_{0} \sin \alpha} \ln \left(k e^{-\frac{c_{0} \sin \alpha}{2}\left(\min _{i=1, \ldots, k} \phi_{i}(x)\right)}\right) \\
& =-\frac{2}{c_{0} \sin \alpha} \ln \left(k e^{-\frac{c_{0} \sin \alpha}{2} \phi^{*}(x)}\right)=\phi^{*}(x)-\frac{2 \ln k}{c_{0} \sin \alpha} .
\end{aligned}
$$

This implies in particular that

$$
-\frac{2 \ln k}{c_{0} \sin \alpha} \leq \phi_{*}-\phi^{*} \leq 0
$$

which shows the first line of (8).

Step 3: Asymptotics (second line of (8)). - We now notice that the set $E_{\infty}$ of edges (where $\phi_{\infty}$ is not $C^{1}$ ) is characterized by

$$
E_{\infty}=\left\{x \in \mathbb{R}^{N-1}, \quad \max _{\nu \in A} x \cdot \nu=x \cdot \nu_{i_{0}}=x \cdot \nu_{i_{1}}, \text { with } \nu_{i_{0}} \neq \nu_{i_{1}} \text { and }\left(\nu_{i_{0}}, \nu_{i_{1}}\right) \in A^{2}\right\} .
$$


For each index $i_{0} \in\{1, \ldots, k\}$, let us denote the convex set

$$
K_{i_{0}}=\left\{x \in \mathbb{R}^{N-1}, \quad x \cdot \nu_{i_{0}}=\max _{\nu \in A} x \cdot \nu\right\} .
$$

Then

$$
\partial K_{i_{0}} \subset \bigcup_{j \neq i_{0}}\left(\nu_{i_{0}}-\nu_{j}\right)^{\perp} .
$$

For $x \in \operatorname{Int}\left(K_{i_{0}}\right)$, let $x_{i_{1}} \in \partial K_{i_{0}} \subset E_{\infty}$ such that

$$
\operatorname{dist}\left(x, E_{\infty}\right)=\left|x-x_{i_{1}}\right| \quad \text { with } \quad x_{i_{1}} \in\left(\nu_{i_{0}}-\nu_{i_{1}}\right)^{\perp} .
$$

For $j \neq i_{0}$, we define the orthogonal projection of $x$ on $\left(\nu_{i_{0}}-\nu_{j}\right)^{\perp}$ as

$$
x_{j}=\operatorname{Proj}_{\mid\left(\nu_{i_{0}}-\nu_{j}\right)^{\perp}}(x) .
$$

In particular $\left|x-x_{j}\right| \geq\left|x-x_{i_{1}}\right|$. Moreover

$$
\begin{aligned}
x \cdot \nu_{j} & =\left(x-x_{j}\right) \cdot \nu_{j}+x_{j} \cdot \nu_{j}=\left(x-x_{j}\right) \cdot \nu_{j}+x_{j} \cdot \nu_{i_{0}} \\
& =\left(x-x_{j}\right) \cdot\left(\nu_{j}-\nu_{i_{0}}\right)+x \cdot \nu_{i_{0}}=x \cdot \nu_{i_{0}}-\left|\nu_{j}-\nu_{i_{0}}\right|\left|x-x_{j}\right| \\
& \leq x \cdot \nu_{i_{0}}-\delta \operatorname{dist}\left(x, E_{\infty}\right)
\end{aligned}
$$

with

$$
\delta=\min _{\nu \neq \nu^{\prime}, \nu, \nu^{\prime} \in A}\left|\nu-\nu^{\prime}\right|>0 .
$$

Therefore

$$
\begin{aligned}
\phi_{*}(x) & =-\frac{2}{c_{0} \sin \alpha} \ln \left(\sum_{i=1}^{k} \lambda_{i} e^{\frac{c_{0} \cos \alpha}{2} x \cdot \nu_{i}}\right) \\
& \geq-\frac{2}{c_{0} \sin \alpha} \ln \left(\sum_{i=1}^{k} \lambda_{i} e^{\frac{c_{0} \cos \alpha}{2}}\left(x \cdot \nu_{i_{0}}-\delta \operatorname{dist}\left(x, E_{\infty}\right)\right)\right.
\end{aligned}
$$

and then for $x \in K_{i_{0}}$, we have

$$
\phi^{*}(x) \geq \phi_{*}(x) \geq \phi^{*}(x)-\frac{2}{c_{0} \sin \alpha} \ln \left(1+\sum_{i \neq i_{0}} \frac{\lambda_{i}}{\lambda_{i_{0}}} e^{\left.-\frac{c_{0} \cos \alpha}{2} \delta \operatorname{dist}\left(x, E_{\infty}\right)\right) .}\right.
$$

This shows that

$$
\lim _{l \rightarrow+\infty} \sup _{\operatorname{dist}\left(x, E_{\infty}\right) \geq l}\left|\phi_{*}(x)-\phi^{*}(x)\right|=0
$$

which implies the second line of (8).

Step 4: Uniqueness. - To end the proof of Theorem 1.2, it only remains to prove uniqueness of the above smooth solution $\phi$ to the mean curvature Equation (3) with the prescribed asymptotics given by $\phi^{*}$. Let $\bar{\phi}$ and $\phi$ be two solutions to (3) with the asymptotics (8). Let

$$
\varepsilon:=\inf \left\{\varepsilon^{\prime}>0 \mid \forall x \in \mathbb{R}^{N-1}, \bar{\phi}(x)+\varepsilon^{\prime} \geq \underline{\phi}(x)\right\}
$$

then for any $x \in \mathbb{R}^{N-1}$,

$$
\bar{\phi}(x)+\varepsilon \geq \underline{\phi}(x)
$$

and there exists a sequence of points $\left(x_{n}\right)_{n}$ such that

$$
\bar{\phi}\left(x_{n}\right)+\varepsilon-\underline{\phi}\left(x_{n}\right) \rightarrow 0 \text { as } n \text { goes to infinity. }
$$


Let us define for any $x \in \mathbb{R}^{N-1}$

$$
\left\{\begin{array}{l}
\bar{\phi}_{n}(x)=\bar{\phi}\left(x+x_{n}\right)-\bar{\phi}\left(x_{n}\right), \\
\underline{\phi}_{n}(x)=\underline{\phi}\left(x+x_{n}\right)-\bar{\phi}\left(x_{n}\right) .
\end{array}\right.
$$

Then, up to the extraction of a subsequence, we have as $n$ goes to infinity

$$
\bar{\phi}_{n} \rightarrow \bar{\phi}_{\infty} \quad \text { and } \quad \underline{\phi}_{n} \rightarrow \underline{\phi}_{\infty}
$$

with a uniform convergence on any compact sets of $\mathbb{R}^{N-1}$. Moreover $\bar{\phi}_{\infty}$ and $\underline{\phi}_{\infty}$ solve Equation (3) and satisfy

$$
\bar{\phi}_{\infty}+\varepsilon \geq \underline{\phi}_{\infty} \quad \text { with equality at } \quad x=0 .
$$

From the strong maximum principle, we deduce that for any $x \in \mathbb{R}^{N-1}$,

$$
\bar{\phi}_{\infty}(x)+\varepsilon=\underline{\phi}_{\infty}(x) .
$$

Let us now assume that $\varepsilon>0$. Because we have

$$
E_{\infty} \subset \bigcup_{\nu \neq \nu^{\prime},\left(\nu, \nu^{\prime}\right) \in A^{2}}\left(\nu-\nu^{\prime}\right)^{\perp}=: \hat{E}_{\infty}
$$

we deduce that there exists $C>0$ such that for any $R \geq 1$ and any $x \in \mathbb{R}^{N-1}$, we have

$$
\sup _{y \in \overline{B_{R}(x)}} \operatorname{dist}\left(y, E_{\infty}\right) \geq \sup _{y \in \overline{B_{R}(x)}} \operatorname{dist}\left(y, \hat{E}_{\infty}\right)=R \sup _{y \in \overline{B_{1}(x / R)}} \operatorname{dist}\left(y, \hat{E}_{\infty}\right) \geq C R
$$

with

$$
C=\inf _{z \in \mathbb{R}^{N-1}}\left(\sup _{y \in \overline{B_{1}(z)}} \operatorname{dist}\left(y, \hat{E}_{\infty}\right)\right) .
$$

We easily check by contradiction that $C>0$. Therefore by (8), let us choose $R$ large enough such that

$$
\sup _{\operatorname{dist}\left(y, E_{\infty}\right) \geq C R}\left|\phi(y)-\phi^{*}(y)\right| \leq \frac{\varepsilon}{4} \quad \text { for } \quad \phi=\bar{\phi}, \underline{\phi} .
$$

Then using (34), we get for some $y_{n} \in \overline{B_{R}\left(x_{n}\right)}$ with $\operatorname{dist}\left(y_{n}, E_{\infty}\right) \geq C R$,

$$
\inf _{y \in B_{R}\left(x_{n}\right)}|\bar{\phi}(y)-\underline{\phi}(y)| \leq\left|\bar{\phi}\left(y_{n}\right)-\underline{\phi}\left(y_{n}\right)\right| \leq\left|\bar{\phi}\left(y_{n}\right)-\phi^{*}\left(y_{n}\right)\right|+\left|\phi^{*}\left(y_{n}\right)-\underline{\phi}\left(y_{n}\right)\right| \leq \frac{\varepsilon}{2} \text {. }
$$

This implies that

$$
\underset{y \in \bar{B}_{R}(0)}{\inf }\left|\bar{\phi}_{\infty}(y)-\underline{\phi}_{\infty}(y)\right| \leq \frac{\varepsilon}{2}
$$

which is in contradiction with (33). Therefore $\varepsilon=0$ and we get $\bar{\phi} \geq \phi$. By symmetry, we also get $\underline{\phi} \geq \bar{\phi}$, which implies $\bar{\phi}=\underline{\phi}$ and shows the uniqueness of the solution. This ends the proof of Theorem 1.2.

\section{Proof of further results in dimension $N=3$}

In this section, the space dimension is $N=3$ and we denote any $x \in \mathbb{R}^{2}$ with its polar coordinates $\left(r, \theta_{x}\right) \in \mathbb{R}^{+} \times[0,2 \pi)$ such that $x=r\left(\cos \theta_{x}, \sin \theta_{x}\right)$. 


\subsection{Classification in dimension $N=3$ of solutions to the eikonal equation with a finite number of singularities}

This subsection gives alternative statement and proof of Proposition 2.3 in dimension $N=3$, in the special case of a finite number of singularities (i.e., gradient jumps).

Proposition 7.1 (Classification with a finite number of singularities, $N=3$ )

Let $\alpha \in\left(0, \frac{\pi}{2}\right], c_{0}>0$ and $c=c_{0} / \sin \alpha$. Choose $\phi_{\infty}$ a 1-homogeneous viscosity solution to the eikonal Equation (10) in dimension $N=3$ with a finite number of singularities on $\mathbb{S}^{1}$. Then the (1) of Theorem 1.3 holds.

Proof of Proposition 7.1. - From Proposition 2.1, we know that there exists a (non empty) compact set $K=\gamma^{-1}(\{0\}) \subset \mathbb{S}^{1}$, such that

$$
\phi_{\infty}(x)=\inf _{\nu \in K}(-(\cot \alpha) \nu \cdot x) .
$$

Thus, for any $\theta \in[0,2 \pi), \psi_{\infty}(\theta)=\phi_{\infty}(\cos \theta, \sin \theta)$ defines a continuous function with values in $[-\cot \alpha, \cot \alpha]$. Firstly $\psi_{\infty \mid K}=-\cot \alpha$. Moreover for any maximal interval $(a, b)$ contained in $\mathbb{S}^{1} \backslash K$, we necessarily have

$$
\psi_{\infty}(\theta)= \begin{cases}-(\cot \alpha) \cos (\theta-a) & \text { if } \quad \theta \in\left[a, \frac{a+b}{2}\right], \\ -(\cot \alpha) \cos (\theta-b) & \text { if } \quad \theta \in\left[\frac{a+b}{2}, b\right] .\end{cases}
$$

Therefore $\phi_{\infty}$ has a singularity (gradient jump) at $\theta=\frac{a+b}{2}$. If $\psi_{\infty}$ only has a finite number of singularities, then we get the characterization of $\psi_{\infty}$ given in the (1) of Theorem 1.3. This ends the proof of Proposition 7.1.

Remark 7.2. - Notice that without assuming that $\phi_{\infty}$ has a finite number of singularities on $\mathbb{S}^{1}$, the set $K$ could be a Cantor set in (35).

Remark 7.3. - Notice that the particular function $\phi_{\infty}(x)=-(\cot \alpha)|x|$ is the analogue (at the level of the eikonal equation) of the level sets of cylindrically symmetric solutions to reaction diffusion equation, constructed in [8] by Hamel, Monneau and Roquejoffre. Similarly, the particular case where the graph of $\phi_{\infty}$ is a pyramid is also the analogue of solutions constructed by Taniguchi in [14].

\subsection{Explicit construction of super-solutions in dimension $N=3$}

In the particular case $N=3$, we construct super-solutions by hand and try to be more precise than in Section 5, above all when $\psi_{\infty}$ is constant and equal to $-(\cot \alpha)$ on some interval $I$. In that case, we construct our super-solution by hand. We explain our ideas on different elementary pieces that we bring together in the proof of Theorem 1.3 to build a global super-solution $\phi^{*}$. Those different elementary pieces are: a cone, an edge or an arc. 


\subsubsection{The cone case}

Lemma 7.4 (Radially symmetric solutions). - Let $\phi_{\infty}$ be the viscosity solution to eikonal Equation (10) whose graph is the straight cone i.e., $\phi_{\infty}(x)=-(\cot \alpha)|x|$ for $x \in \mathbb{R}^{2}$. Then, there exists a unique radially symmetric solution $\phi_{c}$ (unique up to an additive constant) to the forced mean curvature Equation (18), satisfying

$$
\phi_{c}^{\prime}(0)=0 \text { and } \phi_{c}(x)=\phi_{\infty}(x)+o(|x|) .
$$

Moreover $\phi_{c}$ is concave and $\left|D \phi_{c}\right| \leq \cot \alpha$. In the case $\alpha=\pi / 2, \phi_{c}$ is zero (up to an additive constant). Otherwise, as $|x|$ goes to infinity, its asymptotics is more precisely given (up to a constant $C \in \mathbb{R})$ by

$$
\phi_{c}(x)=-(\cot \alpha)|x|+\frac{1}{c_{0} \sin \alpha} \ln |x|+C+\frac{2-3 \sin ^{2} \alpha}{c_{0}^{2} \sin (2 \alpha)|x|}+O\left(\frac{1}{|x|^{2}}\right), \quad \alpha \neq \frac{\pi}{2} .
$$

Moreover, let $\phi_{*}$ be the sub-solution defined by (24) with $\mu=\frac{d \theta}{2 \pi}$ and $N=3$. Fix $\phi_{c}$ such that $C=C_{0}:=\frac{\ln \left(\pi c_{0} \cos \alpha\right)}{c_{0} \sin \alpha}$, then for any $x \in \mathbb{R}^{2}, \phi_{c}(x) \geq \phi_{*}(x)$ and as $|x|$ goes to infinity

$$
\phi_{c}(x)=\phi_{*}(x)+O\left(\frac{1}{\sqrt{|x|}}\right) .
$$

Proof of Lemma 7.4. - This result is proved using quite classical methods. The proof is sketched for the reader's convenience. We also mention that similar results were already obtained by Ninomiya and Taniguchi, see Theorem 3.1 in [12]. With a slight misuse of notation, we denote in the case of radially symmetric solutions $\phi_{c}(x)$ by $\phi_{c}(|x|)=\phi_{c}(r)$ with $r=|x| \geq 0$. Then, Equation (18) reads

$$
-\frac{\phi_{c}^{\prime}}{r}-\frac{\phi_{c}^{\prime \prime}}{1+\phi_{c}^{\prime 2}}+c_{0} \sqrt{1+\phi_{c}^{\prime 2}}-c=0, \quad r>0 .
$$

Thus, $\phi_{c}$ satisfies an ODE involving only its first two derivatives and it can only be defined up to constants. Setting $v=\phi_{c}^{\prime}$, we get

$$
v^{\prime}=\left(1+v^{2}\right)\left(c_{0} \sqrt{1+v^{2}}-c-\frac{v}{r}\right):=\left(1+v^{2}\right) g(v, r), \quad r>0 .
$$

The proof of Lemma 7.4 now reduces to the study of this ODE (existence, uniqueness and asymptotics).

7.2.1.1. Step 1: Existence. - Since for any $r>0, g(0, r) \leq 0$ and $g\left(v_{0}(r), r\right)=0$ where

$$
v_{0}(r)=-\frac{c^{2}-c_{0}^{2}}{\frac{c}{r}+c_{0} \sqrt{\frac{1}{r^{2}}+c^{2}-c_{0}^{2}}} \leq 0,
$$

$v=0$ is a super-solution and $v=v_{0}$ is a negative decreasing sub-solution to the ODE (38). Thus, for every $r_{1}>0$, there exist $r_{2}>r_{1}$ and a solution $v \in C^{\infty}\left(\left(r_{1}, r_{2}\right), \mathbb{R}\right)$ to the ODE $v^{\prime}=\left(1+v^{2}\right) g(v, r)$ satisfying $v_{0} \leq v \leq 0$ for any $r \in\left(r_{1}, r_{2}\right)$. Moreover we have $g_{v}^{\prime}(v, r) \leq 0$ for $v \in\left[v_{0}, 0\right]$, and then we conclude that

$$
v^{\prime} \leq 0 \text { for } \quad r \in\left(r_{1}, r_{2}\right)
$$


7.2.1.2. Step 2: Qualitative properties. - Since for any $r>0, v_{0}(r) \in(-\cot \alpha, 0)$, the bounds of $v(r)$ by $v_{0}(r)$ and zero force $v$ to exist globally for $r>0$. Moreover, as $\lim _{r \rightarrow 0} v_{0}(r)=0, v$ satisfies the same limit and we can extend $v$ to 0 by continuity as $v(0)=0$. This proves that $v$ is a global smooth solution to (38) with initial condition $v(0)=0$. Thus it is easy to check that any primitive function $\phi_{c}$ to $v$ is a smooth radially symmetric solution to (18) satisfying $\phi_{c}^{\prime}(0)=0$. From (39), we conclude that $\phi_{c}$ is concave.

7.2.1.3. Step 3: Asymptotics. - Since $v$ is strictly decreasing on $\mathbb{R}^{+}$and bounded from below, it converges to a finite limit - $\cot \alpha \leq l<0$ as $r$ goes to infinity. Since $v$ is uniformly bounded in $[-\cot \alpha, 0], l$ must satisfy $g(l,+\infty)=0$ which leads to $l=-\cot \alpha$.

Linearising Equation (38) around $-\cot \alpha$, we set $w=v+\cot \alpha$. As $w$ is uniformly bounded on $\mathbb{R}^{+}$and goes to zero at infinity, Equation (38) reads

$$
w^{\prime}(r)=-c(\cot \alpha) w+\tilde{g}(w, r), \quad r>0
$$

where $\tilde{g}(w, r)=O\left(w^{2}\right)+O(1 / r)$ as $r$ goes to infinity. By Duhamel's formula, $w$ follows exponentially fast the behavior of the slowest term of $\tilde{g}$. Thus $w \sim C / r$ as $r$ goes to infinity and a straight calculation gives $C=1 /\left(c_{0} \sin \alpha\right)$. Repeating this method up to order 2 , one gets

$$
v(r)=-\cot \alpha+\frac{1}{c_{0}(\sin \alpha) r}+\frac{3 \sin ^{2} \alpha-2}{c_{0}^{2} \sin (2 \alpha) r^{2}}+O\left(\frac{1}{r^{3}}\right) .
$$

This gives the desired asymptotics for $\phi_{c}$ up to constants.

Step 4: Uniqueness. - Let $\phi_{c}^{1}$ and $\phi_{c}^{2}$ be two smooth radially symmetric solutions to (18). From step 3, we know that they satisfy the same asymptotic expansion as $r$ goes to infinity and we assume the constants $C$ are the same. Since $\phi_{c}^{1}-\phi_{c}^{2}$ solves an elliptic equation with smooth coefficients and no zero order term, the classical maximum principle applies. Hence $\phi_{c}^{1}-\phi_{c}^{2}=0$ because $\lim _{r \rightarrow \infty}\left(\phi_{c}^{1}-\phi_{c}^{2}\right)(r)=0$. This proves the uniqueness of $\phi_{c}$ up to constants.

Step 5: Comparison with $\phi_{*}$. - Using Lemma 8.1, we can check (37) with the suitable value of the constant $C=C_{0}$ (see for instance the computation (45) with $N_{0}(x) \simeq 1 / \sqrt{\pi}$ ). Finally, using the comparison principle (as in Step 4), we deduce that $\phi_{*} \leq \phi_{c}$. This ends the proof of Lemma 7.4.

\subsubsection{The edge case}

Lemma 7.5 (Edge super-solution). - Assume $\phi_{*}$ is given by (24) where the measure $\mu$ is the sum of two Dirac masses

$$
\mu=\mu_{\left\{\theta_{1}, \theta_{2}\right\}}=\lambda_{1} \delta_{\theta_{1}}+\lambda_{2} \delta_{\theta_{2}}
$$

with $\lambda_{i}>0, \theta_{i} \in[0,2 \pi)$ for $i=1,2$ such that $\theta_{1}<\theta_{2}$ and $\delta_{\theta_{i}}$ the Dirac mass in $\theta_{i}$. In the case $\alpha \neq \pi / 2$, define $\phi_{e}$ for any $x \in \mathbb{R}^{2}$ by

$$
\phi_{e}(x)=\min \left(p_{1}(x), p_{2}(x)\right) \text { with } p_{i}(x)=-(\cot \alpha) x \cdot \nu_{i}-\frac{2}{c_{0} \sin \alpha} \ln \lambda_{i}
$$

where $\nu_{i}=\left(\cos \theta_{i}, \sin \theta_{i}\right)$. Then, $\phi_{e}$ is a Lipschitz and piecewise smooth global super-solution to (18) verifying $\phi_{*} \leq \phi_{e}$ on $\mathbb{R}^{2}$. Moreover, as $|x|$ goes to infinity,

$$
\phi_{e}(x)=\phi_{*}(x)+O(1), \quad x \in \mathbb{R}^{2} .
$$

4 e SÉRIE - TOME $46-2013$ - No 2 
Proof of Lemma 7.5. - Notice first that $\phi_{e}=\phi^{*}$ with $\phi^{*}$ defined as a special case of Proposition 5.1. This shows that $\phi_{e}$ is a concave (Lipschitz) supersolution. Finally (41) follows from (32). This ends the proof of the lemma.

7.2.3. The arc case. - Here we wish to describe a super-solution to (18) which, from above, looks like an arc, i.e., is made up of two non parallel straight lines connected by a circle.

Lemma 7.6 (Arc super-solution). - Assume $\phi_{*}$ is given by (24) where the measure $\mu$ is the sum of two Dirac masses and a Lebesgue measure

$$
\mu=\mu_{\left[\theta_{1}, \theta_{2}\right]}=\lambda \delta_{\theta_{1}}+\lambda \delta_{\theta_{2}}+\mathbb{I}_{\left(\theta_{1}, \theta_{2}\right)} \mathrm{d} \theta,
$$

where $\lambda>0, \theta_{i} \in[0,2 \pi)$ for $i=1,2$ and $\theta_{1}<\theta_{2}$.

Define $\phi_{e}$ as in the edge case (40) with $\lambda_{1}=\lambda_{2}=\lambda$. Define $\phi_{c}$ as in the cone case (Lemma 7.4) where the constant $C \in \mathbb{R}$ in (36) is chosen such that $\phi_{c}(0)=\phi_{e}(0)=$ $-\frac{2}{c_{0} \sin \alpha} \ln \lambda$.

Finally, define $\phi_{a}$ on $\mathbb{R}^{2}$ by

$$
\forall x \in \mathbb{R}^{2}, \quad \phi_{a}(x)= \begin{cases}-\frac{2}{c_{0} \sin \alpha} \ln \lambda & \text { if } x=0 \\ \min \left(\phi_{c}(x), \phi_{e}(x)\right) & \text { if } \theta_{x} \in\left(\theta_{1}, \theta_{2}\right) \\ \phi_{e}(x) & \text { otherwise. }\end{cases}
$$

Then $\phi_{a}$ is a Lipschitz continuous global super-solution to (18). Moreover, as $|x|$ goes to infinity

$$
\phi_{a}(x)=\phi_{*}(x)+O(1) .
$$

The shape of $\phi_{a}$ is sketched on Figures 1,2,3.

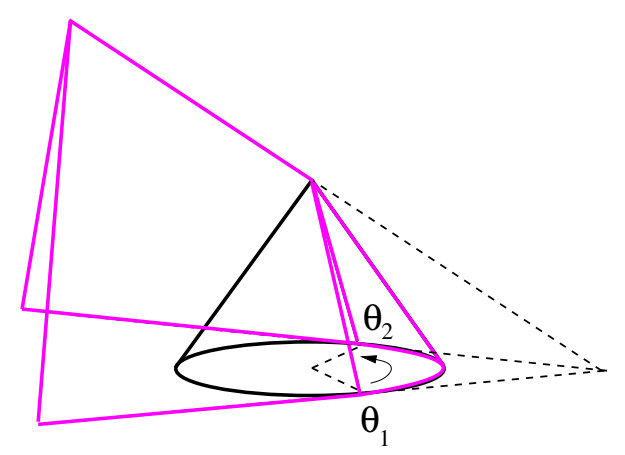

FIGURE 1. Sketch of $\phi_{a}$ for $\theta_{2}-\theta_{1}<\pi$

Proof of Lemma 7.6. - Step 1: $\phi_{a}$ is a global continuous super-solution. - By definition and Lemmas 7.4 and 7.5, $\phi_{a}$ is a super-solution to (18) where it is locally the minimum of supersolutions, i.e., everywhere except on the two half lines $\theta_{x}=\theta_{i}$ for $i=1,2$. However, we have $\phi_{c}(0)=\phi_{e}(0)$ and $D \phi_{e}(x)=-(\cot \alpha) \nu_{i}$ while $\phi_{c}^{\prime}(r) \in(-\cot \alpha, 0]$ for any $x \in \mathbb{R}^{2}$ with $\theta_{x}=\theta_{i}, i=1$ or 2 . Thus, $\phi_{e}(x) \leq \phi_{c}(x)$ on a neighborhood $\mathcal{N}$ (not containing the origin) of the two half lines $\theta_{x}=\theta_{i}$ for $i=1,2$. This implies $\phi_{a}=\phi_{e}$ on $\mathcal{N}$ and then $\phi_{a}$ is at least a supersolution on $\mathbb{R}^{2} \backslash\{0\}$. Moreover, $\phi_{a}$ is a supersolution on the whole $\mathbb{R}^{2}$. Indeed, 


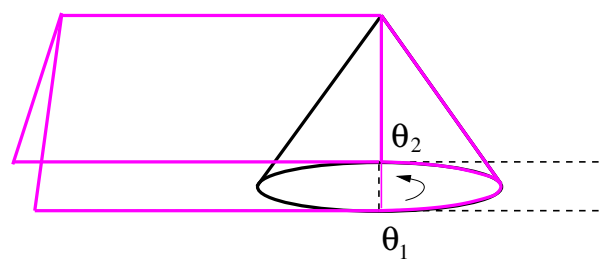

FIGURE 2. Sketch of $\phi_{a}$ for $\theta_{2}-\theta_{1}=\pi$

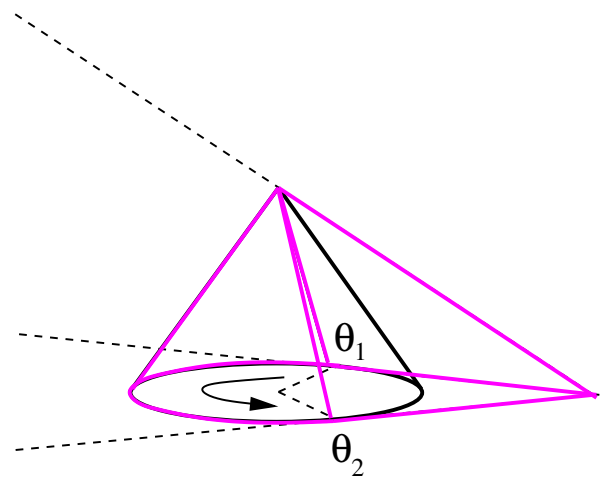

FIGURE 3. Sketch of $\phi_{a}$ for $\theta_{2}-\theta_{1}>\pi$

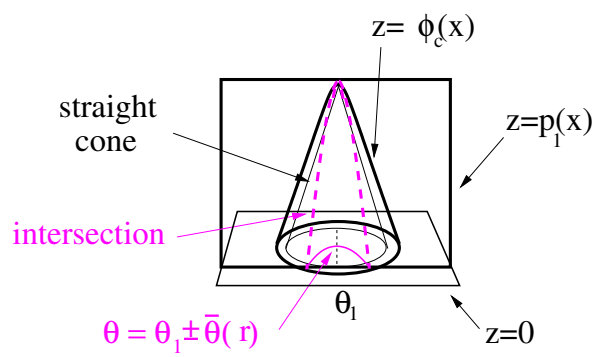

FIGURE 4. Intersection of $z=-(\cot \alpha) v_{1} \cdot x$ with $z=\phi_{c}(x)$

$\phi_{a}=\phi_{e}$ for $\theta_{x} \notin\left[\theta_{1}, \theta_{2}\right]$, then $\phi_{a}$ has a gradient jump along the edge $\theta=\left(\theta_{1}+\theta_{2}\right) / 2+\pi$ up to the origin. And this gradient jump implies that there is no $C^{2}$ test function touching $\phi_{a}$ from below at $x=0$.

Step 2: Relative positions of $\phi_{e}$ and $\phi_{c}$. - Let us now study the relative positions of both graphs of $\phi_{e}$ and $\phi_{c}$. Since $\phi_{c}(x) \in\left(-|x| \cot \alpha+\phi_{c}(0), \phi_{c}(0)\right]$, we have

$$
\begin{aligned}
p_{1}(x)=\phi_{c}(x) & \Longleftrightarrow-r(\cot \alpha) \cos \left(\theta_{x}-\theta_{1}\right)+\phi_{c}(0)=\phi_{c}(r) \\
& \Longleftrightarrow \theta_{x}=\theta_{1} \pm \arccos \left(\frac{\phi_{c}(r)-\phi_{c}(0)}{-r \cot \alpha}\right):=\theta_{1} \pm \bar{\theta}(r)
\end{aligned}
$$


where $\bar{\theta}(r) \in(0, \pi / 2)$ for $r>0$ (see Figure 4). Notice that from the concavity of $\phi_{c}$, we deduce that the set

$$
\left\{x \in \mathbb{R}^{2} \mid p_{1}(x) \leq \phi_{c}(x)\right\}=\left\{x \in \mathbb{R}^{2} \mid \theta_{x} \in\left[\theta_{1}-\bar{\theta}(r), \theta_{1}+\bar{\theta}(r)\right]\right\}
$$

is a convex set. Therefore we deduce that

$$
\phi_{e}(x) \leq \phi_{c}(x) \Leftrightarrow \theta_{x} \in\left[\theta_{1}-\bar{\theta}(r), \theta_{1}+\bar{\theta}(r)\right] \cup\left[\theta_{2}-\bar{\theta}(r), \theta_{2}+\bar{\theta}(r)\right]
$$

and $\phi_{a}(x)=\phi_{c}(x)$ if and only if $\theta_{x} \in I_{r}=\left[\theta_{1}+\bar{\theta}(r), \theta_{2}-\bar{\theta}(r)\right]$. Since we choose $\phi_{c}$ such that $\phi_{c}^{\prime}(0)=0$, we get

$$
\lim _{r \rightarrow 0} \bar{\theta}(r)=+\frac{\pi}{2}
$$

This forces both curves $\theta_{x}=\theta_{1}+\bar{\theta}(r)$ and $\theta_{x}=\theta_{2}-\bar{\theta}(r)$ to intersect at some point $(x, z) \neq\left(0, \phi_{c}(0)\right)$ as soon as $\theta_{2}-\theta_{1}<\pi$. In that case, it is worth noticing that the above interval $I_{r}$ is empty for sufficiently small $r$ (see Figures 5,6 ).

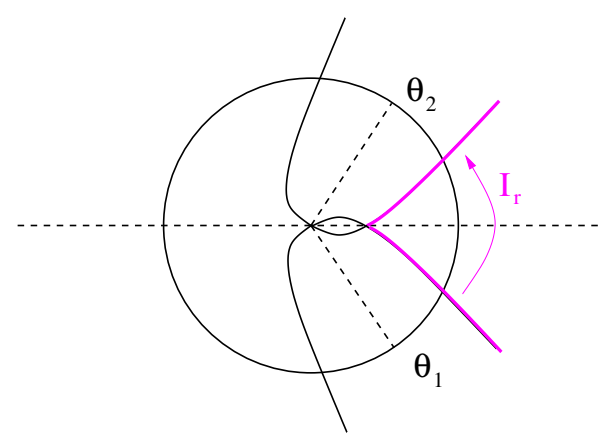

FIGURE 5. The set $I_{r}$ if $\theta_{2}-\theta_{1}<\pi$

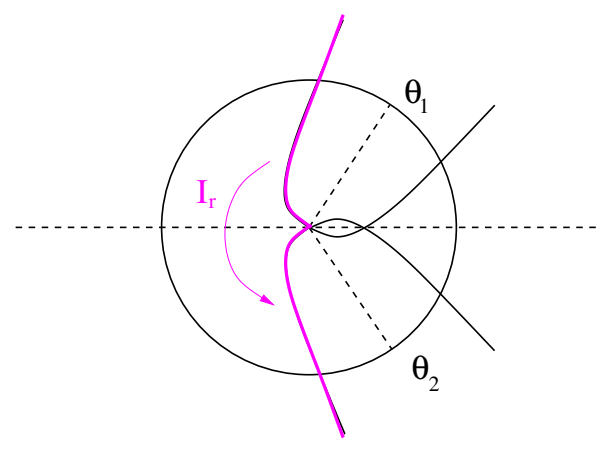

FIGURE 6. The set $I_{r}$ if $\theta_{2}-\theta_{1}>\pi$

On the other hand, using the asymptotics (36) of $\phi_{c}$ found in Lemma 7.4, we get as $r$ goes to infinity

$$
\cos \bar{\theta}(r)=1-\frac{1}{c_{0} \cos \alpha} \frac{\ln r}{r}+\frac{\phi_{c}(0)-C}{r \cot \alpha}+O\left(\frac{1}{r^{2}}\right), \quad \alpha \neq \frac{\pi}{2}
$$

where $C$ is the constant given by (36) and fixed by the choice $\phi_{c}(0)=\phi_{e}(0)$. 
Step 3: $\phi_{*} \leq \phi_{a}+$ constant. - Let $\phi_{*}$ be the sub-solution given by (24) where the measure $\mu$ is $\mu_{\left[\theta_{1}, \theta_{2}\right]}$, i.e., with $b=c_{0} \cos \alpha$ :

$$
\phi_{*}(x)=-\frac{2}{c_{0} \sin \alpha} \ln \left(\lambda e^{\frac{b r}{2} \cos \left(\theta_{x}-\theta_{1}\right)}+\lambda e^{\frac{b r}{2} \cos \left(\theta_{x}-\theta_{2}\right)}+\int_{\theta_{1}}^{\theta_{2}} e^{\frac{b r}{2} \cos \left(\theta_{x}-\theta\right)} d \theta\right) .
$$

Each term in the logarithm being non negative, we have

$$
\phi_{*} \leq \phi_{e} \quad \text { on } \quad \mathbb{R}^{2} .
$$

To prove that $\phi_{a}$ is above the sub-solution $\phi_{*}$ up to an additive constant, it remains to compare $\phi_{*}$ and $\phi_{c}$ when $\theta_{x} \in I_{r}=\left[\theta_{1}+\bar{\theta}(r), \theta_{2}-\bar{\theta}(r)\right]$ and $r$ sufficiently large.

According to Lemma 8.1, one gets that for any $x \in \mathbb{R}^{2}$ for $r=|x|$ sufficiently large and uniformly in $\theta_{x} \in\left[\theta_{1}, \theta_{2}\right]$

$$
\phi_{*}(x)=-(\cot \alpha) r+\frac{\ln r}{c_{0} \sin \alpha}-\frac{2}{c_{0} \sin \alpha} \ln (\Phi(x))
$$

with $\Phi(x):=\frac{2 \pi N_{0}(x)}{\sqrt{b}}+\lambda \sqrt{r} e^{\frac{b r}{2}\left(\cos \left(\theta_{x}-\theta_{1}\right)-1\right)}+\lambda \sqrt{r} e^{\frac{b r}{2}\left(\cos \left(\theta_{x}-\theta_{2}\right)-1\right)}+O\left(\frac{1}{\sqrt{r}}\right)$

where

$$
N_{0}(x)=\int_{\sqrt{r} g\left(\theta_{1}-\theta_{x}\right)}^{\sqrt{r} g\left(\theta_{2}-\theta_{x}\right)} e^{-\frac{u^{2}}{4}} \frac{\mathrm{d} u}{2 \pi}
$$

as defined in Lemma 8.1. Since $g$ is odd and $\theta_{x} \in\left[\theta_{1}, \theta_{2}\right]$, we see that

$$
N_{0}(x) \geq \int_{0}^{\sqrt{r} g\left(\frac{\theta_{2}-\theta_{1}}{2}\right)} e^{-\frac{u^{2}}{4}} \frac{\mathrm{d} u}{2 \pi}=\frac{1}{2 \sqrt{\pi}}+o(1) .
$$

We deduce in particular that for $r$ large enough and uniformly in $\theta_{x} \in\left[\theta_{1}, \theta_{2}\right]$ :

$$
\Phi(x) \geq \frac{\sqrt{\pi}}{2 \sqrt{b}} .
$$

Therefore, from the asymptotics (36) of $\phi_{c}$, we deduce that there exist $r_{1}>0, C_{1}>0$ such that

$$
\forall r \geq 0, \quad \forall \theta_{x} \in\left[\theta_{1}, \theta_{2}\right], \quad r \geq r_{1} \Rightarrow \phi_{*}(x) \leq \phi_{c}(x)+C_{1} .
$$

Now from (44) and (46), we deduce that (up to increasing the constant $C_{1}$ ),

$$
\forall r \geq 0, \quad \forall \theta_{x} \in\left[\theta_{1}, \theta_{2}\right], \quad r \geq r_{1} \Rightarrow \phi_{*}(x) \leq \phi_{a}(x)+C_{1} .
$$

Step 4: $\phi_{*} \geq \phi_{a}-$ constant.

Case 1: $\theta_{x} \in I_{r}$. - We start with the asymptotics (45). Using (43), we see that there exist $r_{2}>0, C_{2}>0$ such that

$\forall i=1,2, \quad \forall r \geq 0, \quad \forall \theta_{x} \in I_{r}, \quad r \geq r_{2} \Rightarrow \sqrt{r} e^{\frac{b r}{2}\left(\cos \left(\theta_{x}-\theta_{i}\right)-1\right)} \leq \sqrt{r} e^{\frac{b r}{2}(\cos (\bar{\theta}(r))-1)} \leq C_{2}$.

Using also the fact that $N_{0}(x) \leq 1 /(2 \sqrt{\pi})$, we deduce that $\Phi$ is bounded for $r$ large enough and then (up to increasing $r_{2}$ and $C_{2}$ )

$$
\forall r \geq 0, \quad \forall \theta_{x} \in I_{r}, \quad r \geq r_{2} \Rightarrow \phi_{*}(x) \geq \phi_{c}(x)-C_{2} .
$$

4 e SÉRIE - TOME $46-2013$ - No 2 
Case 2: $\theta_{x} \in\left[\theta_{1}, \theta_{2}\right] \backslash I_{r}$. - Let us assume that $\theta_{x} \in\left[\theta_{1}, \theta_{1}+\bar{\theta}(r)\right)$ (the symmetric case is similar). Then there exist $r_{3}>0, C_{3}>0$ such that

$\forall r \geq 0, \quad \forall \theta_{x} \in\left[\theta_{1}, \theta_{1}+\bar{\theta}(r)\right), \quad r \geq r_{3} \Rightarrow \sqrt{r} e^{\frac{b r}{2}\left(\cos \left(\theta_{x}-\theta_{1}\right)-1\right)} \geq \sqrt{r} e^{\frac{b r}{2}(\cos (\bar{\theta}(r))-1)} \geq C_{3}>0$.

Therefore (up to increasing the constants $r_{3}$ and $C_{3}$ ), for any $r \geq 0$ and any $\theta_{x} \in\left[\theta_{1}, \theta_{1}+\bar{\theta}(r)\right)$,

$$
r \geq r_{3} \Rightarrow \Phi(x) \leq C_{3}\left(\lambda \sqrt{r} e^{\frac{b r}{2}\left(\cos \left(\theta_{x}-\theta_{1}\right)-1\right)}+\lambda \sqrt{r} e^{\frac{b r}{2}\left(\cos \left(\theta_{x}-\theta_{2}\right)-1\right)}\right)
$$

and then

$$
\begin{aligned}
\phi_{*}(x) & \geq-\frac{2}{c_{0} \sin \alpha} \ln \left\{\lambda e^{\frac{b r}{2} \cos \left(\theta_{x}-\theta_{1}\right)}+\lambda e^{\frac{b r}{2} \cos \left(\theta_{x}-\theta_{2}\right)}\right\}-\frac{2 \ln C_{3}}{c_{0} \sin \alpha} \\
& \geq-\frac{2}{c_{0} \sin \alpha} \ln \left(2 e^{-\frac{c_{0} \sin \alpha}{2} \phi_{e}(x)}\right)-\frac{2 \ln C_{3}}{c_{0} \sin \alpha} \\
& =\phi_{e}(x)-\frac{2 \ln \left(2 C_{3}\right)}{c_{0} \sin \alpha} .
\end{aligned}
$$

Case 3: $\theta_{x} \in \mathbb{S}^{1} \backslash\left[\theta_{1}, \theta_{2}\right]$. - Notice that the set $\mathbb{S}^{1} \backslash\left[\theta_{1}, \theta_{2}\right]$ is not empty because $\theta_{2}-\theta_{1}<2 \pi$ (as a consequence of $\theta_{1}, \theta_{2} \in[0,2 \pi)$ ). In that case, we set

$$
\theta_{m}^{\prime}=\theta_{m}-\pi \quad \text { with } \quad \theta_{m}=\frac{\theta_{1}+\theta_{2}}{2} .
$$

Then, it satisfies $\theta_{2}-2 \pi<\theta_{m}^{\prime}<\theta_{1}$. Let us assume that (the other case is similar):

$$
\theta_{x} \in\left[\theta_{m}^{\prime}, \theta_{1}\right)
$$

We also define

$$
\theta_{x}^{\prime}=\theta_{x}+\pi
$$

Then we have

$$
\int_{\theta_{1}}^{\theta_{2}} e^{\frac{b r}{2} \cos \left(\theta_{x}-\theta\right)} d \theta=\int_{\left[\theta_{1}, \theta_{2}\right] \cap\left[\theta_{x}, \theta_{x}^{\prime}\right]}(\ldots) d \theta+\int_{\left[\theta_{1}, \theta_{2}\right] \backslash\left[\theta_{x}, \theta_{x}^{\prime}\right]}(\ldots) d \theta .
$$

We have

Using (49), we also see that

$$
\int_{\left[\theta_{1}, \theta_{2}\right] \cap\left[\theta_{x}, \theta_{x}^{\prime}\right]} e^{\frac{b r}{2} \cos \left(\theta_{x}-\theta\right)} d \theta \leq \pi e^{\frac{b r}{2} \cos \left(\theta_{x}-\theta_{1}\right)} .
$$

$$
\int_{\left[\theta_{1}, \theta_{2}\right] \backslash\left[\theta_{x}, \theta_{x}^{\prime}\right]} e^{\frac{b r}{2} \cos \left(\theta_{x}-\theta\right)} d \theta \leq \pi e^{\frac{b r}{2} \cos \left(\theta_{x}-\theta_{1}\right)} .
$$

Therefore, we conclude that in this third case,

$$
\begin{aligned}
\phi_{*}(x) & \geq-\frac{2}{c_{0} \sin \alpha} \ln \left\{(\lambda+2 \pi) e^{\frac{b r}{2} \cos \left(\theta_{x}-\theta_{1}\right)}+(\lambda+2 \pi) e^{\frac{b r}{2} \cos \left(\theta_{x}-\theta_{2}\right)}\right\} \\
& \geq \phi_{e}(x)-\frac{2 \ln \left(\frac{2(\lambda+2 \pi)}{\lambda}\right)}{c_{0} \sin \alpha} .
\end{aligned}
$$

Conclusion. - Putting (47), (48) and (50) together, we get that there exists a constant $C>0$ such that for $r$ large enough and uniformly in $\theta_{x} \in \mathbb{S}^{1}$.

$$
\phi_{*}(x) \geq \phi_{a}(x)-C \text {. }
$$


The functions $\phi_{*}$ and $\phi_{a}$ being continuous, the result still holds for any $r \geq 0$ (up to increasing the constant $C$ ). This concludes the proof of Lemma 7.6.

\subsection{Proof of Theorem 1.3}

Step 1: Existence of a solution. - Let $\alpha \in\left(0, \frac{\pi}{2}\right], c_{0}>0$ and $c=c_{0} / \sin \alpha$. The case $\alpha=\pi / 2$ is obvious and we omit it. Choose $\phi_{\infty}$ a 1 -homogeneous viscosity solution to the eikonal Equation (10) in dimension $N=3$ with a finite number $m$ of singularities. By Proposition 7.1, the (1) of Theorem 1.3 is already established, and we can consider the measure $\mu$ given in the (2) of Theorem 1.3. Then Proposition 4.3 implies that the function $\phi_{*}$ given by (9) is a smooth concave subsolution of (18).

If $k=1, \phi_{\infty}$ has no gradient jump and the corresponding measure is $\mu=\mathrm{d} \theta$ or $\mu=\lambda_{0}\left(\delta_{\theta_{1}}+\delta_{\theta_{1}+2 \pi}\right)$. In the first case, we saw in Lemma 7.4 that $\phi_{c}$, to which a suitable constant is added, is a smooth solution to (18) with the right asymptotics at infinity. In the second one, $\phi=\phi_{\infty}$ is a suitable solution to (18).

We now turn to the case $k \geq 2$. For any $i \in\{1, \ldots, k\}$, choose $\lambda_{0}>0$ a given positive constant. We have

$$
\mu=\sum_{j} \mu_{j} \geq \tilde{\mu}_{i}
$$

with

$$
\tilde{\mu}_{i}=2 \lambda_{0}\left(\delta_{\theta_{i}}+\delta_{\theta_{i+1}}\right)+\sigma_{i} \mathbb{I}_{\left(\theta_{i}, \theta_{i+1}\right)} \mathrm{d} \theta .
$$

Let $\tilde{\phi}_{i *}$ be the subsolution defined in (24) with the measure $\tilde{\mu}_{i}$. If $\tilde{\mu}_{i}$ corresponds to an arc $\left(\sigma_{i}=1\right)$, denote $\tilde{\phi}_{i}^{*}$ the global supersolution defined in Lemma 7.6 with $\lambda=2 \lambda_{0}$. Notice that there is a constant $C>0$ (that can be chosen independently of the index $i$ ) such that

$$
\tilde{\phi}_{i}^{*}-C \leq \tilde{\phi}_{i *} \leq \tilde{\phi}_{i}^{*}+C \text {. }
$$

If $\tilde{\mu}_{i}$ corresponds to an edge $\left(\sigma_{i}=0\right)$, denote $\tilde{\phi}_{i}^{*}$ the global supersolution defined in Lemma 7.5 with $\lambda_{1}=\lambda_{2}=2 \lambda_{0}$, which satisfies in particular (52). Finally, define on $\mathbb{R}^{2}$ the function $\tilde{\phi}^{*}$ as the infimum over $i \in\{1, \ldots, k\}$ of $\tilde{\phi}_{i}^{*}$. Notice that, by construction, we have

$$
\tilde{\phi}^{*}(x)=\tilde{\phi}_{i}^{*}(x) \quad \text { if } \quad \theta_{x} \in\left[\theta_{i}, \theta_{i+1}\right] .
$$

We also have in particular

$$
\phi_{*} \leq \tilde{\phi}_{i *} \leq \tilde{\phi}_{i}^{*}+C \leq \tilde{\phi}^{*}+C=: \phi^{*} .
$$

We claim that at infinity

$$
\phi^{*}(x)=\phi_{*}(x)+O(1) .
$$

We shall first finish the proof of Theorem 1.3 and come back to the proof of that claim in a second step. Thus $\phi^{*}$ is a global supersolution above the subsolution $\phi_{*}$. Moreover, either there exists $\sigma_{i}=1$ and then we have (see in particular Figures 1,2,3)

$$
\text { there exists } p \in \mathbb{R}^{N-1} \text { such that } \limsup _{|x| \rightarrow+\infty} \frac{\phi^{*}(x)-p \cdot x}{|x|}<0 \text {. }
$$

Or $\sigma_{i}=0$ for any $i$, and condition (55) is satisfied if $k \geq 3$. The special case $k=2$ and $\sigma_{1}=\sigma_{2}=0$ corresponds to an edge for which we already know the existence of a smooth 
concave solution, by Theorem 1.2. In the other cases, condition (55) and Proposition 3.1 imply the existence of a smooth concave solution $\phi \in\left[\phi_{*}, \phi^{*}\right]$.

Step 2: Proof of (54) in the case $k \geq 2$. - Let $x \in \mathbb{R}^{2}$, then there exists $i \in\{1, \ldots, k\}$ such that $\theta_{x} \in\left[\theta_{i}, \theta_{i+1}\right]$.

We can write:

where $\tilde{\mu}_{i}$ is defined by (51). So

$$
\mu=\tilde{\mu}_{i}+\bar{\mu}_{i}
$$

$$
\operatorname{supp}\left(\bar{\mu}_{i}\right) \subset \mathbb{S}^{1} \backslash\left(\theta_{i}, \theta_{i+1}\right) \quad \neq \varnothing .
$$

Then $k \geq 2$ implies that

$$
l_{x}:=\min \left\{\theta_{x}-\theta_{i}, \theta_{i+1}-\theta_{x}\right\}<\pi
$$

and

$$
\int_{\mathbb{S}^{1}} e^{\frac{b r}{2} \cos \left(\theta_{x}-\theta\right)} d \bar{\mu}_{i}(\theta) \leq \bar{\mu}_{i}\left(\mathbb{S}^{1}\right) e^{\frac{b r}{2} \cos l_{x}} \leq \frac{\bar{\mu}_{i}\left(\mathbb{S}^{1}\right)}{2 \lambda_{0}} \int_{\mathbb{S}^{1}} e^{\frac{b r}{2} \cos \left(\theta_{x}-\theta\right)} d \tilde{\mu}_{i}(\theta)
$$

with

$$
\int_{\mathbb{S} 1} e^{\frac{b r}{2} \cos \left(\theta_{x}-\theta\right)} d \tilde{\mu}_{i}(\theta)=2 \lambda_{0} e^{\frac{b r}{2} \cos \left(\theta_{x}-\theta_{i}\right)}+2 \lambda_{0} e^{\frac{b r}{2} \cos \left(\theta_{x}-\theta_{i+1}\right)}+\sigma_{i} \int_{\theta_{i}}^{\theta_{i+1}} e^{\frac{b r}{2} \cos \left(\theta_{x}-\theta\right)} \mathrm{d} \theta .
$$

Therefore we have

$$
\begin{aligned}
\phi_{*}(x) & \geq-\frac{2}{c_{0} \sin \alpha} \ln \left\{\left(1+\frac{\bar{\mu}_{i}\left(\mathbb{S}^{1}\right)}{2 \lambda_{0}}\right) \int_{\mathbb{S}^{1}} e^{\frac{b r}{2} \cos \left(\theta_{x}-\theta\right)} d \tilde{\mu}_{i}(\theta)\right\} \\
& =\tilde{\phi}_{i *}(x)-\frac{2}{c_{0} \sin \alpha} \ln \left(1+\frac{\bar{\mu}_{i}\left(\mathbb{S}^{1}\right)}{2 \lambda_{0}}\right) \\
& \geq \tilde{\phi}_{i}^{*}(x)-C^{\prime}
\end{aligned}
$$

where we have used (52) in the last line. Using (53), we see that this implies

$$
\phi_{*}(x) \geq \phi^{*}(x)-C^{\prime \prime} \text { for } \theta_{x} \in\left[\theta_{i}, \theta_{i+1}\right] .
$$

Finally, this implies (54) and ends the proof of Theorem 1.3.

\section{Appendix: Laplace's method}

For the reader's convenience, we reproduce here Laplace's method. It investigates asymptotics as $r$ goes to infinity of integrals involving expressions of the form $e^{-r J}, J$ denoting some given function. Our interest is to find uniform estimates as $x=r\left(\cos \theta_{x}, \sin \theta_{x}\right)$ lies in a given angle sector $\left[\theta_{1}, \theta_{2}\right]$. The proof develops ideas that can be found for a simpler case in [4], Chapter 4.5.2 page 204. Lemma 8.1 below is only used in Steps 3 and 4 of the proof of Lemma 7.6.

Lemma 8.1 (Uniform asymptotics in a sector $\left[\theta_{1}, \theta_{2}\right]$ ). - Define for any $x=r\left(\cos \theta_{x}, \sin \theta_{x}\right) \in \mathbb{R}^{2}$ with $\theta_{x} \in[0,2 \pi)$

$$
F(x)=\lambda_{1} e^{\frac{b r}{2} \cos \left(\theta_{1}-\theta_{x}\right)}+\lambda_{2} e^{\frac{b r}{2} \cos \left(\theta_{2}-\theta_{x}\right)}+\int_{\theta_{1}}^{\theta_{2}} e^{\frac{b r}{2} \cos \left(\theta-\theta_{x}\right)} f(\theta) \frac{\mathrm{d} \theta}{2 \pi}
$$

where 
(i) $b=c_{0} \cos \alpha>0, \lambda_{i} \in \mathbb{R}, \theta_{i} \in[0,2 \pi]$ for $i=1,2$ and $\theta_{1}<\theta_{2}$

(ii) $f \in C^{1}([0,2 \pi], \mathbb{C})$ is $2 \pi$-periodic.

As $r$ goes to infinity, we have the following asymptotics uniform in the angular sector $\theta_{x} \in\left[\theta_{1}, \theta_{2}\right]$

$$
F(x)=\lambda_{1} e^{\frac{b r}{2} \cos \left(\theta_{1}-\theta_{x}\right)}+\lambda_{2} e^{\frac{b r}{2} \cos \left(\theta_{2}-\theta_{x}\right)}+e^{\frac{b r}{2}}\left(\frac{f\left(\theta_{x}\right)}{\sqrt{b r}} N_{0}(x)+\frac{R(x)}{r}\right)
$$

where

and

$$
N_{0}(x)=\int_{\sqrt{r} g\left(\theta_{1}-\theta_{x}\right)}^{\sqrt{r} g\left(\theta_{2}-\theta_{x}\right)} e^{-\frac{u^{2}}{4}} \frac{\mathrm{d} u}{2 \pi} \quad \in[0,1 / \sqrt{\pi}]
$$

$$
g(\theta)=\left\{\begin{array}{lll}
\operatorname{sign}(\theta) \sqrt{2 b(1-\cos \theta)} & \text { for } & \theta \in[-\pi, \pi] \\
\operatorname{sign}(\theta) 2 \sqrt{b} & \text { for } \quad \theta \in \mathbb{R} \backslash[-\pi, \pi] .
\end{array}\right.
$$

Moreover, there exists a constant $C>0$ such that for any $x \in \mathbb{R}^{2}$, if $r>1$ and $\theta_{x} \in\left[\theta_{1}, \theta_{2}\right]$ then $|R(x)| \leq C$.

Proof of Lemma 8.1. - It is straightforward to check that $g$ defined by (58) is an odd $C^{3}$-diffeomorphism from $[-\pi, \pi]$ to $[-2 \sqrt{b}, 2 \sqrt{b}]$ satisfying $g(0)=0, g^{\prime}(0)=\sqrt{b}$ and $g^{\prime \prime}(0)=0$. We have also chosen to extend $g$ to the real line by continuity. However, when we speak about $g^{-1}$, it has to be understood as the inverse of $g$ on $[-\pi, \pi]$.

Afterwards, for any $x \in \mathbb{R}^{2}$, we define

$$
I(x):=\int_{\theta_{1}}^{\theta_{2}} e^{\frac{b r}{2} \cos \left(\theta-\theta_{x}\right)} f(\theta) \frac{\mathrm{d} \theta}{2 \pi}=\int_{\theta_{1}-\theta_{x}}^{\theta_{2}-\theta_{x}} e^{\frac{b r}{2} \cos \theta} f\left(\theta+\theta_{x}\right) \frac{\mathrm{d} \theta}{2 \pi} .
$$

Assume $\theta_{x} \in\left[\theta_{1}, \theta_{2}\right]$. In order to get a bound on $I$ uniform in the angle $\theta_{x}$, we fix some $\delta>0$ and set

$$
\theta_{*}=\left\{\begin{array}{ll}
\theta_{1}-\theta_{x} & \text { if } \theta_{1}-\theta_{x} \geq-\pi+\delta \\
-\pi+\delta & \text { otherwise }
\end{array} \theta^{*}= \begin{cases}\theta_{2}-\theta_{x} & \text { if } \theta_{2}-\theta_{x} \leq \pi-\delta \\
\pi-\delta & \text { otherwise }\end{cases}\right.
$$

We then cut the integral $I$ into three parts, integrating between $\theta_{1}-\theta_{x}$ and $\theta_{*}$, between $\theta_{*}$ and $\theta^{*}$ and finally between $\theta^{*}$ and $\theta_{2}-\theta_{x}$. We call those three integrals $I_{1}, I_{2}$ and $I_{3}$ respectively.

Regarding $I_{1}$ and $I_{3}, \cos \theta$ can be bounded in both cases by $\cos (\pi-\delta)$ and $f$ by its $L^{\infty}$ norm on the compact set $[0,2 \pi]$. Thus, there exists a constant $C>0$ such that for any $x \in \mathbb{R}^{2}$ with $\theta_{x} \in\left[\theta_{1}, \theta_{2}\right]$,

$$
I_{1}+I_{3} \leq C e^{\frac{b r}{2} \cos (\pi-\delta)} .
$$

For sufficiently small $\delta>0$, the right hand term decreases exponentially fast and the contribution of $I_{1}$ and $I_{3}$ in $I$ is exponentially small as $r$ goes to infinity uniformly in $\theta_{x}$.

Using the change of variables $u=\sqrt{r} g(\theta)$, we rewrite $I_{2}$ as

$$
I_{2}(x)=\frac{e^{\frac{b r}{2}}}{\sqrt{b r}} \int_{\sqrt{r} g\left(\theta_{*}\right)}^{\sqrt{r} g\left(\theta^{*}\right)} e^{-\frac{u^{2}}{4}} h\left(\frac{u}{\sqrt{r}}\right) \frac{\mathrm{d} u}{2 \pi}
$$

where $h(t)=f\left(\theta_{x}+g^{-1}(t)\right) / \sqrt{1-\left(t^{2} /(4 b)\right)}$. Since $h(t)=h(0)+\int_{0}^{t} h^{\prime}(s) \mathrm{d} s$, we have

$$
I_{2}(x)=e^{\frac{b r}{2}}\left(\frac{f\left(\theta_{x}\right)}{\sqrt{b r}} N_{0}^{*}(x)+\frac{R(x)}{r}\right)
$$


where $N_{0}^{*}$ is defined as in Lemma 8.1 with $\theta_{*}$ or $\theta^{*}$ when needed, but it only changes the desired asymptotics with an exponentially small correction as above. The remainder term $R$ is defined as

$$
R(x)=r^{\frac{1}{2}} \int_{\sqrt{r} g\left(\theta_{*}\right)}^{\sqrt{r} g\left(\theta^{*}\right)} e^{-\frac{u^{2}}{4}} \int_{0}^{\frac{u}{\sqrt{r}}} \frac{h^{\prime}(s)}{\sqrt{b}} \mathrm{~d} s \frac{\mathrm{d} u}{2 \pi} .
$$

Since $h$ is smooth, $h^{\prime}$ is uniformly bounded on $\left[g\left(\theta_{*}\right), g\left(\theta^{*}\right)\right]$ and the bound only depends on $\delta$. A straight calculation then shows that there exists $C>0$ such that

$$
R(x) \leq C \int_{\sqrt{r} g\left(\theta_{*}\right)}^{\sqrt{r} g\left(\theta^{*}\right)}|u| e^{-\frac{u^{2}}{4}} \mathrm{~d} u \leq C \int_{-\infty}^{+\infty}|u| e^{-\frac{u^{2}}{4}} \mathrm{~d} u .
$$

Putting finally $I_{1}, I_{2}$ and $I_{3}$ together, we get the desired asymptotics.

\section{REFERENCES}

[1] G. Barles, H. M. Soner, P. E. Souganidis, Front propagation and phase field theory, SIAM J. Control Optim. 31 (1993), 439-469.

[2] L. A. Caffarelli, W. Littman, Representation formulas for solutions to $\Delta u-u=0$ in $\mathbf{R}^{n}$, in Studies in partial differential equations, MAA Stud. Math. 23, Math. Assoc. America, 1982, 249-263.

[3] M. G. Crandall, H. Ishit, P.-L. Lions, User's guide to viscosity solutions of second order partial differential equations, Bull. Amer. Math. Soc. (N.S.) 27 (1992), 1-67.

[4] L. C. Evans, Partial differential equations, Graduate Studies in Math. 19, Amer. Math. Soc., 1998.

[5] P. C. FIFE, Dynamics of internal layers and diffusive interfaces, CBMS-NSF Regional Conference Series in Applied Mathematics 53, Society for Industrial and Applied Mathematics (SIAM), 1988.

[6] D. Gilbarg, N. S. Trudinger, Elliptic partial differential equations of second order, Grundl. Math. Wiss. 224, Springer, 1977.

[7] F. Hamel, R. Monneau, J.-M. Roquejoffre, Existence and qualitative properties of multidimensional conical bistable fronts, Discrete Contin. Dyn. Syst. 13 (2005), 1069-1096.

[8] F. Hamel, R. Monneau, J.-M. Roquejoffre, Asymptotic properties and classification of bistable fronts with Lipschitz level sets, Discrete Contin. Dyn. Syst. 14 (2006), 75-92.

[9] C. Imbert, Convexity of solutions and $C^{1,1}$ estimates for fully nonlinear elliptic equations, J. Math. Pures Appl. 85 (2006), 791-807.

[10] J. I. KANEL', Stabilization of solutions of the Cauchy problem for equations encountered in combustion theory, Mat. Sb. (N.S.) 59 (101) (1962), 245-288.

[11] P. De Mottoni, M. Schatzman, Geometrical evolution of developed interfaces, Trans. Amer. Math. Soc. 347 (1995), 1533-1589.

[12] H. Ninomiya, M. TAniguchi, Traveling curved fronts of a mean curvature flow with constant driving force, in Free boundary problems: theory and applications, I (Chiba, 1999), GAKUTO Internat. Ser. Math. Sci. Appl. 13, Gakkōtosho, 2000, 206-221. 
[13] H. Ninomiya, M. Taniguchi, Existence and global stability of traveling curved fronts in the Allen-Cahn equations, J. Differential Equations 213 (2005), 204-233.

[14] M. TANiguchi, Multi-dimensional traveling fronts in bistable reaction-diffusion equations, Discrete Contin. Dyn. Syst. 32 (2012), 1011-1046.

(Manuscrit reçu le 2 juillet 2011;

accepté, après révision, le 13 septembre 2012.)

\author{
Régis Monneau \\ Université Paris-Est \\ École des Ponts ParisTech \\ CERMICS \\ 6-8, av. B. Pascal \\ Cité Descartes \\ 77455 Marne-La-Vallée Cedex 2, France \\ E-mail: monneau@cermics.enpc.fr \\ Jean-Michel RoqueJofFre \\ Institut de Mathématiques de Toulouse \\ (UMR CNRS 5219) \\ Université Paul Sabatier \\ 118 route de Narbonne \\ 31062 Toulouse Cedex 4, France \\ E-mail: jean-michel.roquejoffre@math.univ-toulouse.fr \\ Violaine ROUSSIER-MICHON \\ Institut de Mathématiques de Toulouse \\ (UMR CNRS 5219) \\ INSA Toulouse \\ 135 avenue de Rangueil \\ 31077 Toulouse Cedex 4, France \\ E-mail: violaine.roussier-michon@math.univ-toulouse.fr
}

